\title{
Experimental Investigation on the Dynamic Responses of a Free-hanging Water Intake Riser under Vessel Motion
}

\author{
Jungao Wang ${ }^{\mathrm{a}, \mathrm{b}}$, Sherry Xiang ${ }^{\mathrm{c}}$, Shixiao $\mathrm{Fu}^{\mathrm{a}, \mathrm{b}^{*}}$, Peimin $\mathrm{Cao}^{\mathrm{c}}$, Jianmin Yang ${ }^{\mathrm{a}, \mathrm{b}}$, Jingxi \\ $\mathrm{He}^{\mathrm{c}}$

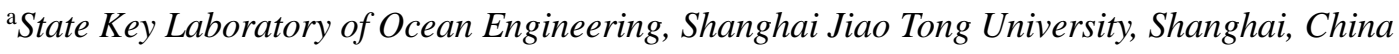 \\ ${ }^{\mathrm{b}}$ Collaborative Innovation Center for Advanced Ship and Deep-Sea Exploration, Shanghai Jiao \\ Tong University, Shanghai, China \\ ${ }^{\mathrm{c}}$ SBM offshore, USA
}

\begin{abstract}
A large-scale model test of a free-hanging water intake riser (WIR) is performed in an ocean basin to investigate the riser responses under vessel motion. Top end of the WIR is forced to oscillate at given vessel motion trajectories. Fiber Brag Grating (FBG) strain sensors are used to measure the WIR dynamic responses. Experimental results firstly confirms that the free-hanging WIR would experience out-of-plane vortex-induced vibrations (VIVs) under pure vessel motion even for the case with a $K C$ number as low as 5. Meanwhile, comparison between numerical results and experimental measurements suggests a significant drag amplification by out-of-plane vessel motioninduced VIV. What's more, further study on WIR response frequencies and cross section trajectories reveals a strong correlation between vessel motion-induced VIV and local $K C$ number distribution, owing to the small $K C$ number effect. The presented work provides useful references for gaining a better understanding on VIV induced by vessel motion, and for the development of future prediction models.
\end{abstract}

Keywords: Vortex-Induced Vibration, small $K C$ number, Oscillatory Flow, Water Intake Riser, Vessel Motion

* Corresponding author. Tel: +86 13501947087 ; Fax: +86 21 34207050-2031 ; E-mail address: shixiao.fu@ sjtu.edu.cn 


\section{Introduction}

As oil and natural gas exploration and production extend to increasingly deep waters, new offshore production concepts have been developed recently including the floating liquefied natural gas (FLNG) system [1]. To improve the FLNG process efficiency and also for the economical consideration, free-hanging water intake risers (WIRs) are proposed to pump up cold water from the sea bottom to the vessel deck for the cooling process and power systems. Ocean currents, vessel motion-induced riser global responses, high internal flow velocities and large diameter (up to several meters) are the major design issues for the WIRs.

When it comes to the ocean current, vortices will be generated in the wake of the riser. The alternate vortex shedding would lead the riser to oscillate in both cross-flow (CF) and in-line (IL) directions, which are called vortex-induced vibrations. VIV may lead to rapid accumulation of fatigue damage of the riser system, and amplify drag forces of the riser systems. These issues have been studied in depth over the last three decades [2-9].

Vessel motion is the other external load for the riser systems. Currently, riser global responses under vessel motion can be numerically calculated by most of the riser analysis software like OrcaFlex [10], Riflex [11] and etc. The uncertainties of these numerical simulations are mostly caused by the insufficient understanding on the hydrodynamic loads [12], for example, drag amplification by VIV [13], and nonlinear problems like riser-soil interaction [14]. Luis [15] conducted a large scale field test on WIR connected to a barge, he reported the modal frequency dependent pipe added mass coefficients were in the range from 0.26 to 0.78 . Recently, Halkyard [16] conducted a WIR model test in an ocean basin, which mainly focused on the riser global dynamic responses under vessel motion. However, other WIR responses, such as internal flow effect and vortex induced vibrations were not addressed.

Recent research also indicates that in addition to the ocean current-induced VIV and vessel motion induced riser global motion, vessel motion can also lead to significant out-of-plane VIV to the compliant risers $[17,18,19,20]$. This phenomenon occurs because the riser will be exposed to the oscillatory flow due to its relative motion with respect to the water particles around. According to the experimental investigations, vessel motion-induced VIV for the compliant risers is characterized with strong time- 
varying features, which is recognized as one of the major differences compared to VIV under ocean current [20]. Meanwhile, maximum KC number and maximum in-plane velocity are found to be the two dominant parameters governing vessel motion-induced VIV features when maximum KC number is large enough (larger than 40 typically [20]). As to the WIR, the $K C$ number along the riser under vessel motion is much smaller thanks to its large diameter. However, considering its bottom boundary condition as a free end, the WIR could possibly have a considerable global in-plane motion along the entire riser. This brings up the question whether the WIR also experience vessel motioninduced VIV under a small $K C$ number scenario? Besides, the internal flow further adds more uncertainty to this problem.

To evaluate the hydrodynamic performance of the WIR under vessel motion and considering the internal flow effect, a large scale model test was carried out for SBM offshore in the ocean basin at Shanghai Jiao Tong University [21, 22]. The scopes of the model test were to measure the dynamic responses of the riser under vessel motion; to investigate the internal flow effect; to observe the occurrence of VIV and axial instability due to vessel motion and / or internal flow. The aim of the test cases reported in this paper is to preliminarily investigate the relationship between the vessel motion, WIR in-plane global motion and out-of-plane VIV responses.

\section{WIR Model Test}

\subsection{Model test design}

The prototype WIR has a large outer diameter at around $4 \mathrm{~m}$ with the water depth at around $1000 \mathrm{~m}$. The Froude similarity is applied as the scaling law to obtain the geometry, weight and vessel motion (KC number) for the scaled model. The Cauchy similarity is applied as the scaling law to obtain the structural properties like axial stiffness and bending stiffness. Considering the maximum water depth of the test basin pit is $40 \mathrm{~m}$, the WIR is designed to have a scaling factor at 26 , and the corresponding designed physical properties at model scale are listed in Table 1. Since the prototype WIR has a very small wall thickness $(\mathrm{t} / \mathrm{D}=1 / 40)$, it's impossible to find a material that satisfies this aspect ratio and keep the structural integrity at the same time. We have tried our best in balancing the designed values and also the manufacturing feasibility. Generally speaking, the as-built model has a larger axial and bending stiffness 
compared to the designed values. This may lead to a higher natural frequency of the asbuilt model, and therefore, the riser response mode may be underestimated in the model test. Meanwhile, the Reynolds number is also much lower in the model test, but with the scaling methodology we adopt in this paper, we have kept the controlling parameter of vessel motion-induced VIV such as KC number and riser motion velocity follow the scaling law. Therefore, we are supposed to observe quite similar VIV response as what should happen in the real case scenario. It should be mentioned that the prototype WIR may not respond to exactly the same hydrodynamic responses as the test scaled model, but the observations are useful to better understand and predict the full-scaled riser.

Table 1: Physical properties of the tested WIR

\begin{tabular}{cccc}
\hline \hline Item & Prototype & $\begin{array}{c}\text { Model Scale } \\
\text { (Designed) }\end{array}$ & $\begin{array}{c}\text { Model Scale } \\
\text { (Measured) }\end{array}$ \\
\hline Total Length $(m)$ & 1000 & 38.46 & 35.66 \\
Outer Diameter $(m)$ & 4 & 0.154 & 0.165 \\
Inner Diameter $(m)$ & 3.9 & 0.15 & 0.15 \\
Mass in Air $(\mathrm{Kg} / m)$ & 1069 & 1.54 & 3.61 \\
Bottom Clump Weight $(\mathrm{Kg})$ & 181530 & 10.08 & 13.53 \\
Bending Stiffness $E I\left(N \cdot \mathrm{m}^{2}\right)$ & $1.72 \mathrm{E} 10$ & 1412.34 & 12022 \\
Tensile Stiffness $E A(N)$ & $8.68 \mathrm{E} 9$ & 481810 & $3.3 \times 10^{6}$ \\
\hline \hline
\end{tabular}

\subsection{Experimental setup}

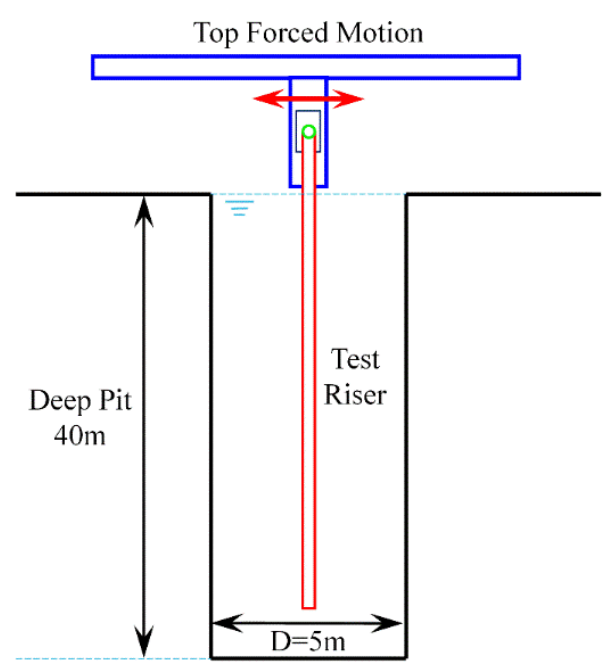


Figure 1 briefly illustrates the WIR model test setup, the deep pit is used to satisfy the designed riser configuration. The top end of the model is pin-connected to a forced motion apparatus, to simulate the vessel motion. It should be noted that there are no background ocean current generated during this model test, the only excitation to the WIR is the prescribed vessel motion, as illustrated in Figure 1.

Detailed view of the top end connection is shown in Figure 2(a), where the test riser was firstly held by a gimbal system (one meter above water surface) which allows the riser to rotate freely in both in-plane and out-of-plane directions. The gimbal system is then rigidly fixed to a steel frame which is connected to the slider of the vertical track. In order to perform the tests with internal flow, there is a flexible hose connected the top of the WIR to a centrifugal pump. In addition, there are two load cells between the gimbal and the WIR to measure the top axial tension as pointed out in Figure 2(a). 


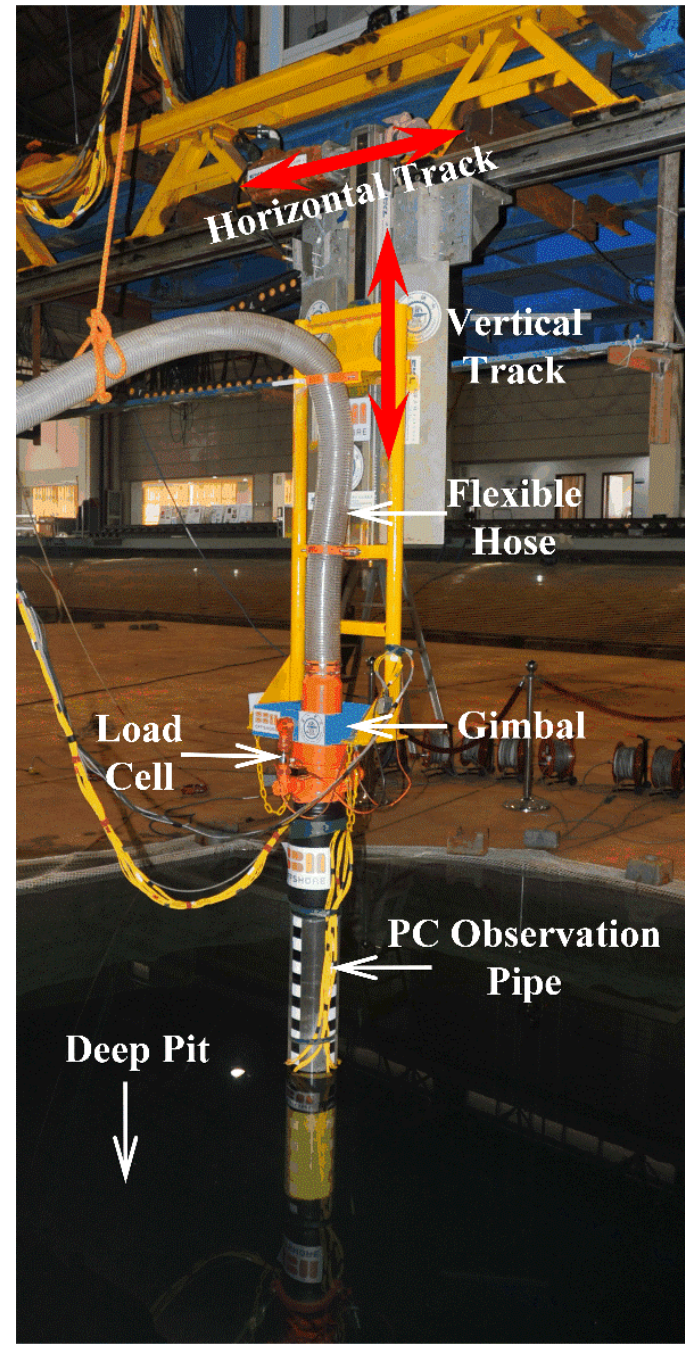

(a)

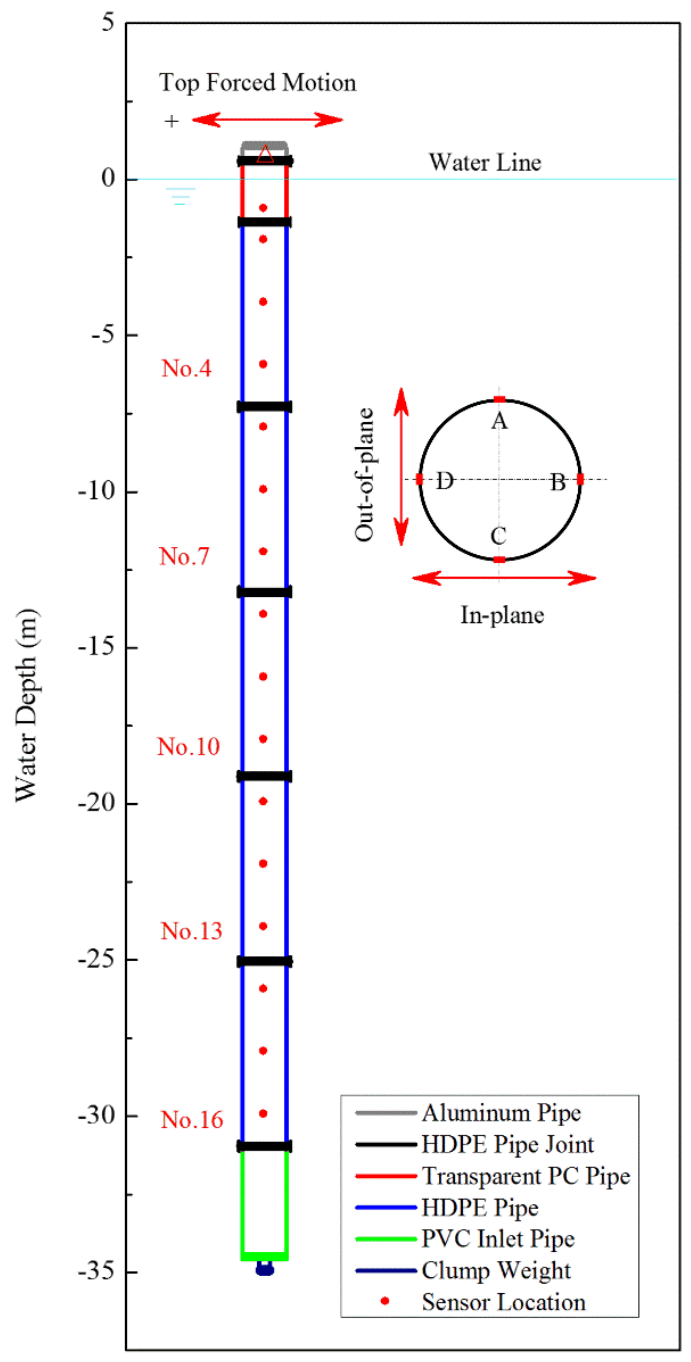

(b)

Figure 2: Detailed view of the WIR model test (a. top forced motion facility and top end connection; b. WIR model configuration and strain sensor layout)

Figure 2(b) presents the configuration of the whole WIR model, including 5 main components from the top to the bottom:

1) A short aluminum pipe (gray part in Figure 2(b)) connected to the gimbal system through a clamp;

2) A transparent Poly Carbonate (PC) pipe (red part in Figure 2(b)) segment used as the observation window;

3) Five High-Density Poly Ethylene (HDPE) pipes (blue parts in Figure 2(b)); 
4) A Poly Vinyl Chloride (PVC) inlet pipe (green part in Figure 2(b)) with an antiswirl device inside;

5) A HDPE cap and clump weight.

All these components are connected by the HDPE sleeve joints (black parts in Figure 2(b)).

Figure 2(b) also demonstrates the Fiber Brag Grating (FBG) strain sensor layout along the entire WIR. There are 16 measuring stations in total. At each measuring station, there are four sensors around the circumference of the section: two for the in-plane responses, and the other two for the out-of-plane responses. The first measuring station is on the PC pipe and it is $0.96 \mathrm{~m}$ below the water line. The second station is $1 \mathrm{~m}$ below the first one, and the rest 14 stations are with the same distance of $2 \mathrm{~m}$ in between.

\subsection{Test matrix}

200 test cases are carried out during the entire test campaign, and they are classified into four different groups in terms of different internal flow rates. Inside of each test group, there are four different vessel motion types including pure horizontal sinusoidal motion, pure vertical sinusoidal motion, circular motion and random motion as summarized in Table 2.

Table 2: WIR model test campaign

\begin{tabular}{|c|c|c|c|}
\hline Group No. & $\begin{array}{c}\text { Internal flow rate } \\
\qquad(\mathrm{L} / \mathrm{min})\end{array}$ & Flexible hose & Vessel Motion \\
\hline 1 & 0 & No & $\begin{array}{c}\text { Horizontal; Vertical; } \\
\text { Circular; Random }\end{array}$ \\
\hline 2 & 0 & Yes & $\begin{array}{c}\text { Horizontal; Vertical; } \\
\text { Circular; Random }\end{array}$ \\
\hline 3 & 500 & Yes & $\begin{array}{l}\text { Horizontal; Vertical; } \\
\text { Circular; Random }\end{array}$ \\
\hline 4 & 1000 & Yes & $\begin{array}{c}\text { Horizontal; Vertical; } \\
\text { Circular; Random }\end{array}$ \\
\hline
\end{tabular}

As we mentioned that vessel motion-induced VIV is the major concern of this paper. 
Therefore, to exclude the potential influence from the internal flow and to have a more basic understanding on vessel motion-induced VIV for the free-hanging WIR, we only select five horizontal sinusoidal motion test cases in Group 1 for analysis as summarized in Table 3. Results and discussion for the other test cases will be carried out separately.

Table 3: Cases for analysis in this paper

\begin{tabular}{ccccccc}
\hline \hline Case No. & $\boldsymbol{A}_{\text {im }}(\boldsymbol{m})$ & $\boldsymbol{T}_{\text {im }}(\boldsymbol{s})$ & $\boldsymbol{f}_{\text {im }}(\mathrm{Hz})$ & $\boldsymbol{V}_{\text {im }}(\boldsymbol{m} / \boldsymbol{s})$ & $\boldsymbol{K} \boldsymbol{C}_{\text {top }}$ & $\boldsymbol{R e}$ \\
\hline 1 & 0.67 & 7.13 & 0.14 & 0.59 & 25.5 & $9.7 \mathrm{E}+04$ \\
2 & 0.59 & 4.75 & 0.21 & 0.78 & 22.5 & $1.3 \mathrm{E}+05$ \\
3 & 0.26 & 3.17 & 0.32 & 0.52 & 9.9 & $8.5 \mathrm{E}+04$ \\
4 & 0.14 & 2.38 & 0.42 & 0.37 & 5.33 & $6.1 \mathrm{E}+04$ \\
5 & 0.06 & 1.58 & 0.63 & 0.24 & 2.28 & $3.9 \mathrm{E}+04$ \\
\hline \hline
\end{tabular}

In Table 3, $A_{\text {im }}$ represents the displacement amplitude of the top forced motion; $T_{i m}$ represents the period of the top forced motion; $f_{i m}$ represents the frequency of the top forced motion; $V_{\text {im }}$ represents the velocity amplitude of the top forced motion; and $K C_{\text {top }}$ represents the $K C$ number at the top end of the WIR, calculated by

$$
K C_{\text {top }}=\frac{2 \pi A_{i m}}{D}
$$

where $D$ is the outer diameter of the WIR.

Re represents the Reynolds number based on the top motion velocity amplitude, calculated by

$$
\mathrm{Re}=\frac{V_{i m} D}{v}
$$

where $V$ is the kinematic viscosity of the water, which is about $10^{-6} \mathrm{~m}^{2} / \mathrm{s}$.

\section{Analysis Procedures}

\subsection{Pre-processing}

In the model test, both in-plane and out-of-plane riser responses are measured using strain sensors. Along the in-plane direction, the measured strain contains four parts: 
initial axial strain, varied axial strain due to global riser motion, varied bending strain caused by global riser motion and varied bending strain caused by VIV. In this paper, only in-plane bending strain is considered, which is calculated by

$$
\varepsilon_{I L}(s, t)=\left(\varepsilon_{I L_{-} D}(s, t)-\varepsilon_{I L_{-} B}(s, t)\right) / 2
$$

where $\varepsilon_{I L_{-} B}(s, t)$ and $\varepsilon_{I L_{-} D}(s, t)$ denote the original strain time series sampled at locations $\mathrm{B}$ and $\mathrm{D}$, as shown in Figure 2. $\varepsilon_{I L}(s, t)$ in Eq. (3) includes bending strain from both in-plane global motion and in-plane VIV.

Meanwhile, pure out-of-plane VIV strain $\varepsilon_{C F_{-} V I V}(s, t)$ after eliminating the influence of tension variations, can be calculated by

$$
\varepsilon_{C F_{-} V V}(s, t)=\left(\varepsilon_{C F_{-} A}(s, t)-\varepsilon_{C F_{-} C}(s, t)\right) / 2
$$

where $\varepsilon_{C F_{-} A}(s, t)$ and $\varepsilon_{C F_{-} C}(s, t)$ denote the original strain time series sampled at locations A and C, as shown in Figure 2.

\subsection{Motion reconstruction method}

Motion reconstruction is based on the assumption that the riser out-of-plane displacement could be expressed as a sum of mode-shapes with different modal weights at any time step:

$$
y(t, s)=\sum_{i=1}^{n} p_{i}(t) \varphi_{i}(s), \quad s \in[0, l]
$$

where $s$ denotes the axial position along the model, $p_{i}(t)$ is the $i^{\text {th }}$ modal weight of displacement, and $\varphi_{i}(s)$ is the $i^{\text {th }}$ mode-shape of the displacement. The modal shapes of the test WIR model are calculated using FEM. The first 6 natural modes of displacement are shown in Figure 3, and the first 6 natural frequencies are shown in Figure 4. Considering the geometrical symmetry of the WIR model, the in-plane and out-of-plane natural frequencies and mode shapes for the same mode are identical. 


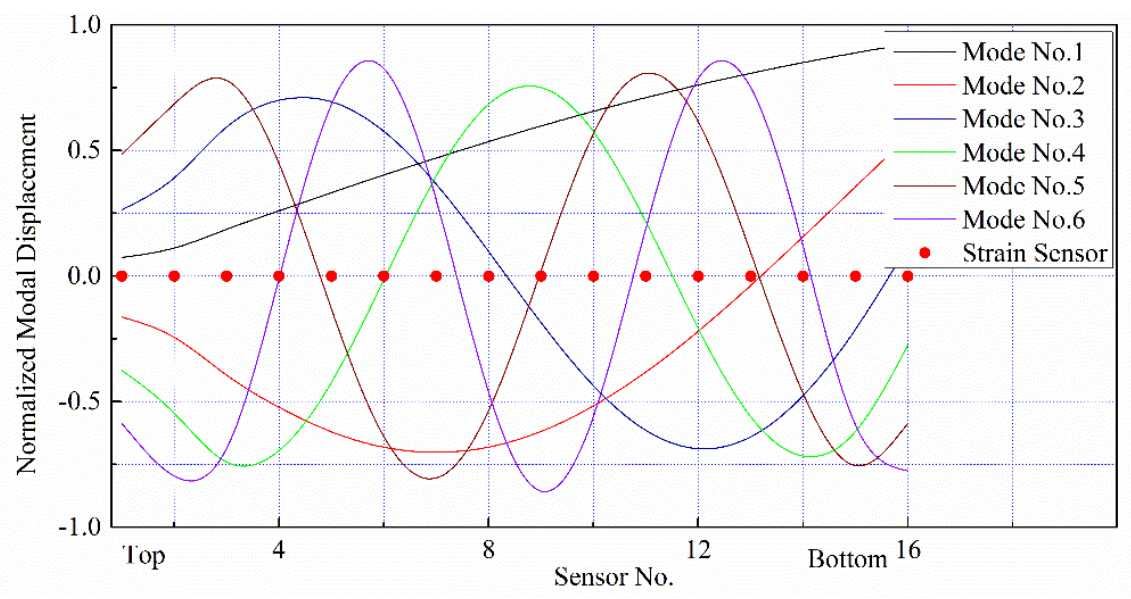

Figure 3 First six natural modes of displacement for the test WIR

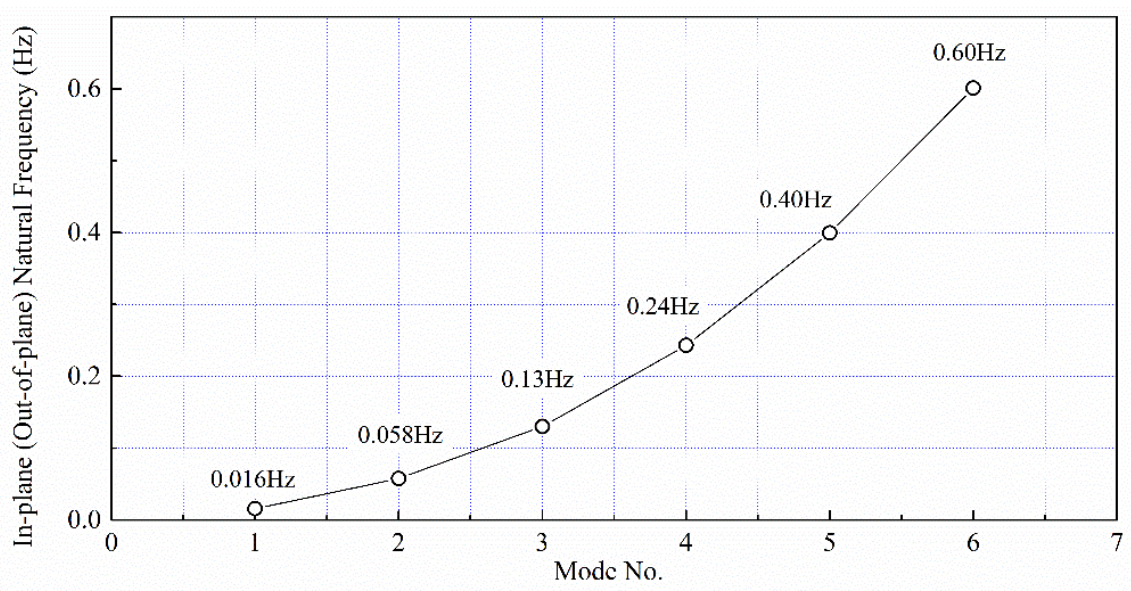

Figure 4 First six natural frequencies for the test WIR in still water

Based on the small deformation assumption, the curvature $\kappa(t, s)$ can be expressed by:

$$
\kappa(t, s)=\frac{d^{2} y}{d s^{2}}=\sum_{i=1}^{n} p_{i}(t) \varphi_{i}^{\prime \prime}(s)
$$

where $\varphi_{i}^{\prime \prime}(s)$ is the $i^{\text {th }}$ mode-shape of the curvature.

According to the geometric relationship between bending strain and curvature, we have

$$
\kappa(t, s)=\frac{\varepsilon(t, s)}{R}
$$


where $R$ is the outer radius of the sensor location on the model.

By combing Eq. (6) and Eq. (7), the out-of-plane displacements can be reconstructed from Eq. (5).

\subsection{Time-frequency analysis}

Under horizontal vessel motion, the WIR will experience equivalent oscillatory flow due to its motion in still water. This equivalent oscillatory flow varies periodically in both amplitude and direction and also varies along the WIR. Considering the interaction between the oscillatory flow and the WIR, the instant shedding frequency can be estimated by

$$
f_{s t}(t, s)=\frac{S t \cdot\left|V_{n}(t, s)\right|}{D}
$$

where $V_{n}(t, s)$ is the in-plane motion velocity that normal to the central axis of the riser model. It should be noted that although Eq. (8) is only applicable to the shedding frequency of a fixed cylinder since the actual shedding frequency of a vibrating cylinder in water will be influenced by the riser vibration. However, it is acceptable to use an equivalent Strouhal number to estimate the shedding frequency distribution. It has been proved that the dominant frequency of vessel motion-induced VIV follows the Strouhal relationship against the maximum in-plane equivalent current velocity with an equivalent Strouhal number $S t=0.14$ [20]. Therefore, we use this value to estimate the shedding frequency of vessel motion-induced VIV in this paper. It should be noted that in the earlier vessel motion-induced VIV model tests, the maximum $K C$ number for all test cases is above 30 . However, for the WIR model test, the $K C$ number will be much smaller. Therefore, in this paper, we should address the question whether the dominant frequency of vessel motion-induced VIV under small $K C$ number follow the Strouhal relationship with $\mathrm{St}=0.14$ as well?

Considering the presence of the time-varying shedding frequency, vessel motioninduced VIV is also expected to be time-varying. Note that this could be the major reason causing the difference between VIV induced by vessel motions and that by steady ocean current according to the published literature [20]. Thus, we introduce the wavelet transformation to represent the time-frequency feature of the out-of-plane VIV 
responses. The continuous wavelet transform equation is expressed as Eq. (9):

$$
W T_{f}(a, \tau)=\left\langle f(t), \psi_{a, \tau}(t)\right\rangle=a^{-1 / 2} \int_{-\infty}^{+\infty} f(t) \psi^{*}\left(\frac{t-\tau}{a}\right) d t
$$

where $W T_{f}(a, \tau)$ is the wavelet transformation coefficient of the time domain signal $f(t)$, which represents the variation of frequency at that time scale. Parameter $a$ is the scale factor, $\tau$ is the shift factor and $\psi(t)$ is the mother wavelet. In this paper, we choose the Morlet wavelet equation as the mother wavelet, which can be defined as

$$
\psi(t)=\mathrm{C} e^{-t^{2} / 2} \cos (5 t)
$$

\section{Results and Discussion}

In the following sections, the riser in-plane global motion due to vessel motion is firstly studied to reveal the equivalent oscillatory flow characteristics along the WIR. Out-ofplane VIV responses including response strain, displacement $\mathrm{A} / \mathrm{D}$, motion trajectories and time-frequency results are further presented. All these topics are discussed only using the case No.1 as an example, more general conclusions concerning all the five cases are introduced in the general discussion part.

\subsection{Numerical WIR in-plane global motion}

Owing to the fact that there were no direct riser displacement measurements in both riser planes during the model test, numerical simulation is performed using OrcaFlex to calculate the in-plane global dynamic responses (displacement amplitude and normal velocity distribution) of the riser under pure vessel motion [10]. The purpose of this numerical simulation is to generate an equivalent current profile based on the riser inplane motion, in order to be able to interpret results later on.

Considering the fact that the real riser motion in the model test can be different from the numerical results partly because VIV is known to amplify the drag forces [13], therefore, four different drag coefficients are used in the numerical simulation to find out the drag coefficient under which the riser has the most similar responses to the experimental measurements.

For the numerical model in OrcaFlex, all the riser physical properties described in Table 1 are modeled. The boundary condition of the riser is pinned at the top and free at the 
bottom. There are 1783 line elements for the entire riser model. The added mass coefficient is set to be a constant at 1 , while the drag coefficient is varied for the purpose of approximation to the experimental measurements. But it should be noted that in the model test, the drag coefficient may vary along the riser as well. During the numerical simulation, a prescribed vessel motion time history is applied at the top end of the riser. For each numerical case, a total 20 vessel motion periods are simulated, and the time step is set to be $0.001 \mathrm{~s}$.

Figure 5(a) presents the standard deviation of the in-plane bending strain comparison among the experimental measurements and numerical simulation results considering different drag coefficients. The comparison indicates three major conclusions:

1) Generally speaking, at the region from the top to sensor No.4, the RMS strain increases with the increasing drag coefficient; but it decreases with the increasing drag coefficient at the rest of the riser (below No.4). This is because the drag force has a phase difference of $180^{\circ}$ with respect to the riser in-plane motion velocity, and it in fact acts as a damping load to the moving WIR. Therefore, the strain in the lower section will be smaller since the increasing drag restricted the riser in-plane motion. At the same time, the motion at the upper part of the riser is also restrained, this is because the top excitation remains the same regardless different drag coefficient, which leads to a larger local curvature (therefore a larger strain) for the case with small local motion (large drag coefficient case).

2) The numerical results marked by the red squares when $C_{D}=1.2$ (1.2 is a conventional drag coefficient used in hydrodynamic response calculation for slender structures) deviate the most from the measured strain (marked by the black dots in Figure 5(a). The best match between the experimental and numerical results is the case when $C_{D}=1.8$, as marked by the green triangles in Figure 5(a). But there are still some discrepancies, which could be partly caused by the drag coefficient variation along the WIR. Nevertheless, this emphasizes the significant contribution of the drag amplification from out-of-plane vessel motion-induced VIV. Drag amplification is proved to be dependent on the out-of-plane VIV displacement amplitude, and Vandiver has proposed an empirical formula estimating drag amplification considering different VIV responses [13]. A larger drag occurs because the current 'sees' a larger projected area due to the riser out-of-plane vibration. 
3) For vessel motion-induced VIV, further analysis will be carried out to identify the accurate drag coefficient distribution considering VIV effects, the corresponding empirical formula is also expected to described drag amplification by vessel motioninduced VIV. These work are essential to the design and analysis for marine slender structures and will be discussed separately in future publication.

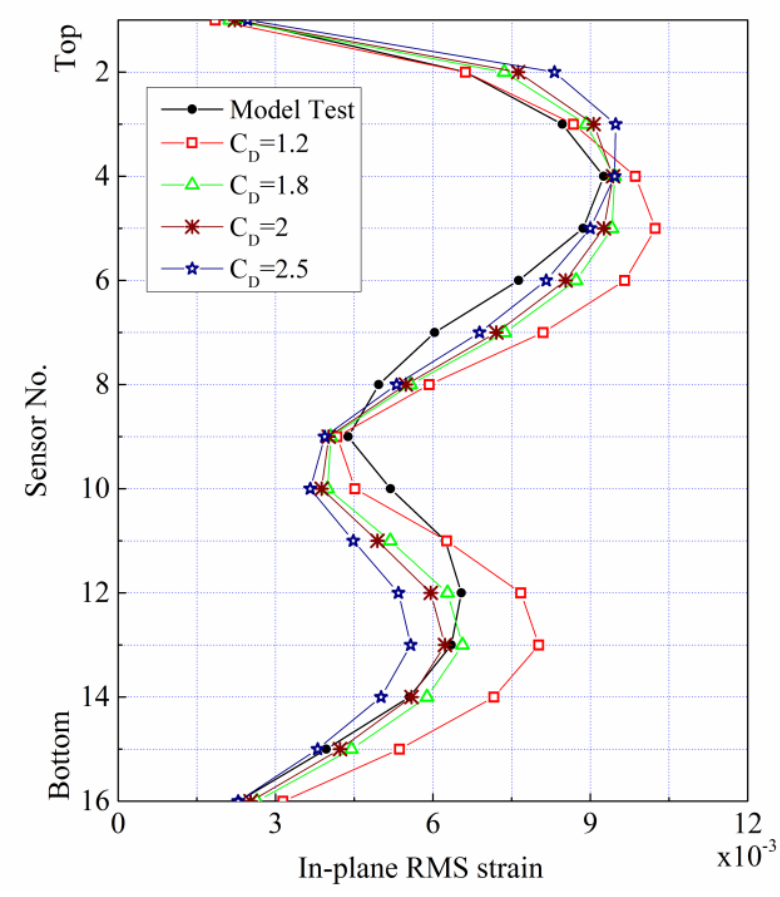

(a)

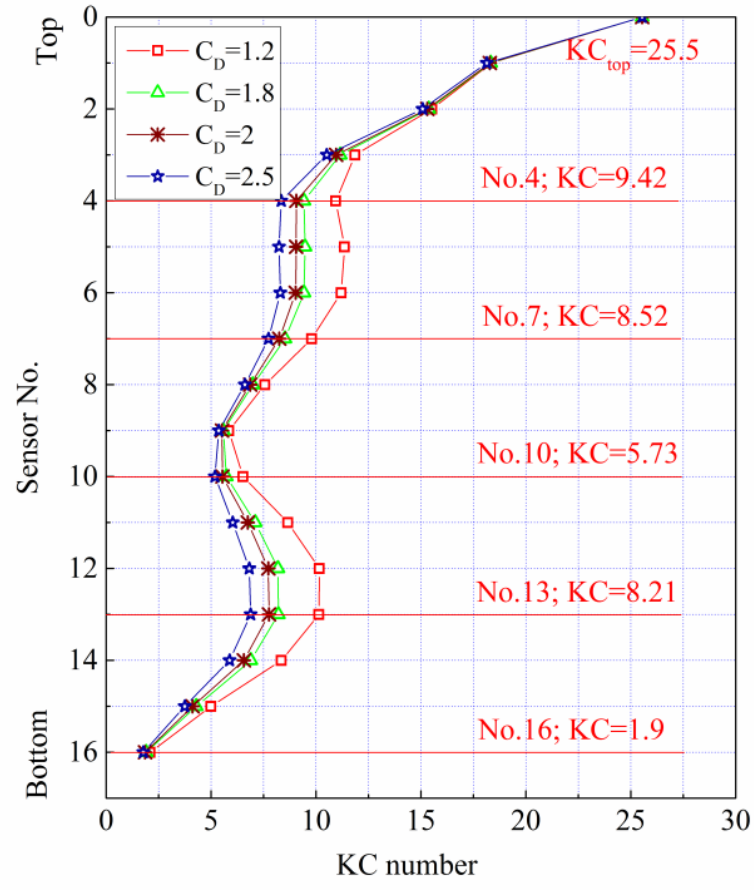

(b)

Figure 5: In-plane global motion results based on numerical simulations (a. in-plane RMS strain comparison between experimental and numerical results under different drag coefficients; $b$. inplane $K C$ number distribution comparison for cases under different drag coefficients)

Figure 5(b) presents the local $K C$ number distribution according to Eq. (11):

$$
K C(s)=\frac{2 \pi A_{n}(s)}{D}
$$

where $A_{n}$ is the displacement amplitude that normal to the riser axsis, and $D$ is the outer diameter of the WIR.

Based on the conclusion in Figure 5(a), $K C$ number distribution when $C_{D}=1.8$ is considered as the best approximation against the experimental results. It can be seen from Figure 5(b) that the $K C$ number decreases sharply below the top excitation point 
due to the hydrodynamic damping loads. Local $K C$ number values at five different measuring stations (No.4; No.7; No.10; No.14; No.16) are given in Figure 5(b), where all of them are smaller than 10 . These relatively small $K C$ number ranges are mostly a result of the large diameter of the WIR, because the corresponding prototype vessel horizontal motion amplitude for this case is about $16.5 \mathrm{~m}$, which is already quite large. This further points out that vessel motion-induced VIV for the WIR is basically characterized with small $K C$ number range, which will be further explained in the flowing sections.

In addition to the $K C$ number distribution, the instantaneous in-plane normal velocity (in other words, the equivalent current velocity profile; the velocity includes the vessel motion period effects) of the riser is the other important aspect to understand vessel motion-induced VIV since it has been unveiled that oscillatory flow-induced VIV was quite 'intermittent' because of the time-varying velocity [20]. Figure 6 presents the calculated space-time varying in-plane normal velocity when $C_{D}=1.8$. It can be seen from Figure 6(a) that the whole horizontal motion period can be divided into two identical phases: the riser is firstly forced to move from the left to the right and then reverse back as marked by the arrows. The contour plot in Figure 6(b) indicates a highly sheared flow profile from the top to the bottom. Besides, as marked in Figure 6(b) when $\mathrm{t}=56.1 \mathrm{~s}$, the instantaneous normal velocity has different current incident angles along the WIR (different color means different incident angle, the current comes from the left to the right at both ends of the WIR, but it comes from the right to the left in the middle part of the WIR). Such instantaneous equivalent current profiles have barely been investigated in the previous current-induced VIV studies. 


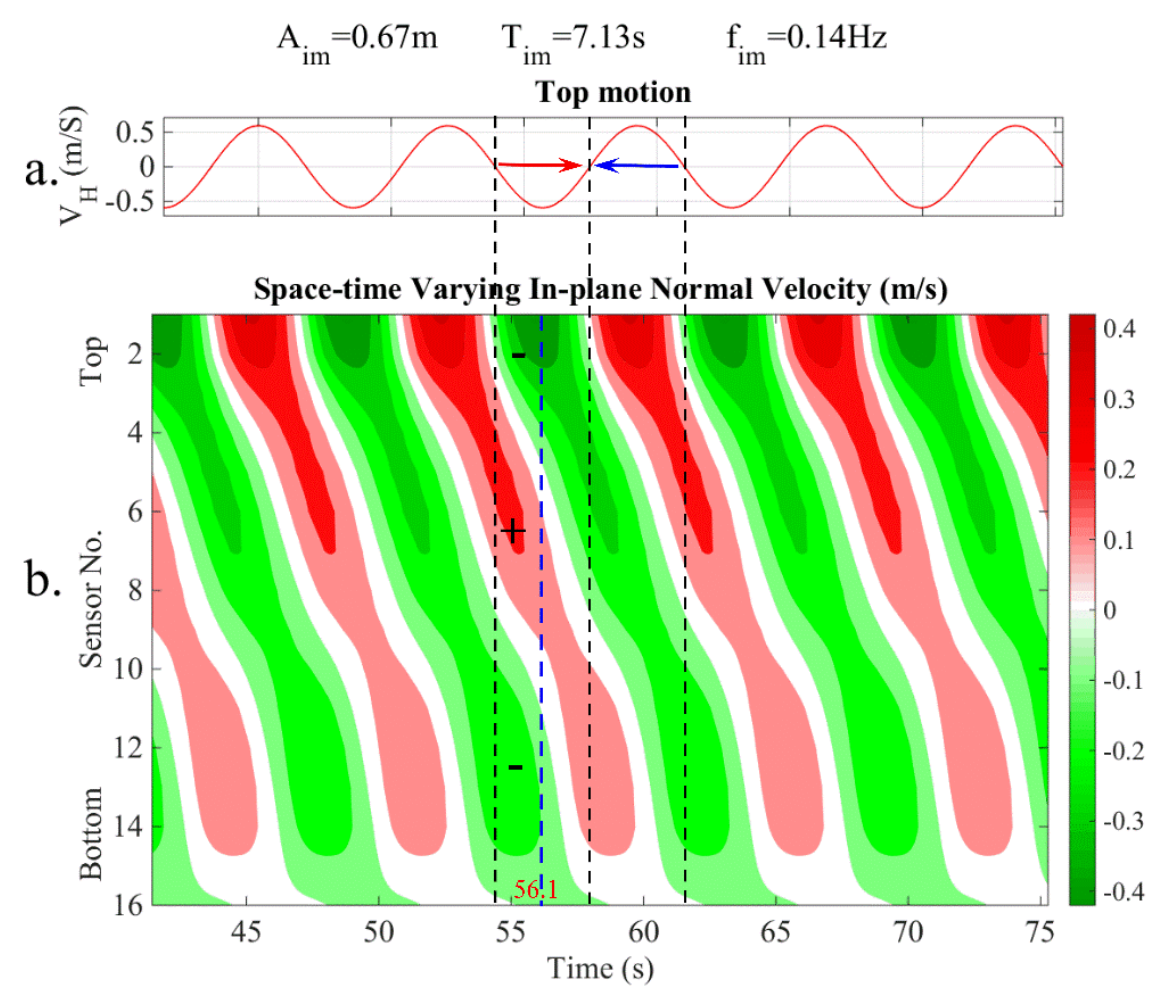

Figure 6: Estimated instantaneous in-plane normal velocity distribution of case $\left(A_{\text {im }}=0.67 \mathrm{~m}\right.$, $T_{i m}=7.13 s$ ) (a: top motion velocity; b: calculated instantaneous normal velocity when $C_{D}=1.8$ )

\subsection{Measured WIR Statistical Responses}

Based on the in-plane and out-of-plane strain measurements along the WIR, Figure 7 presents the statistical strain distribution at both directions in terms of RMS value, as presented in Figure 7(a)), and the maximum and minimum values are presented in Figure 7(b). In Figure 7(b), the maximum and minimum out-of-plane strain distributions do not look as symmetric as the in-plane strains. This is supposed to be related to two aspects: 1) the random nature of VIV;2) considering a very small local $K C$ number, the VIV responses are a bit chaotic due to the small $K C$ number effect (The vortices from the previous motion period are still active during the next period, which may also contribute to the steady response for the small $K C$ number cases. Similar observations were also reported for VIV under small $K C$ number oscillatory flow [20].) Comparison in Figure 7 further illustrates that the in-plane global responses are much significant than the out-of-plane vibration responses in terms of strain amplitude. However, the envelop curves further illustrate a higher out-of-plane response mode (mode $4,0.24 \mathrm{~Hz}$ ) compared to that along the in-plane direction (mode 3,0.13Hz). This 
suggests that the fatigue damage contributed by out-of-plane vibration should be comparable to that along the in-plane direction. Therefore, fatigue damage caused by out-of-plane vessel motion-induced vibration should also be additionally considered in future WIR design.
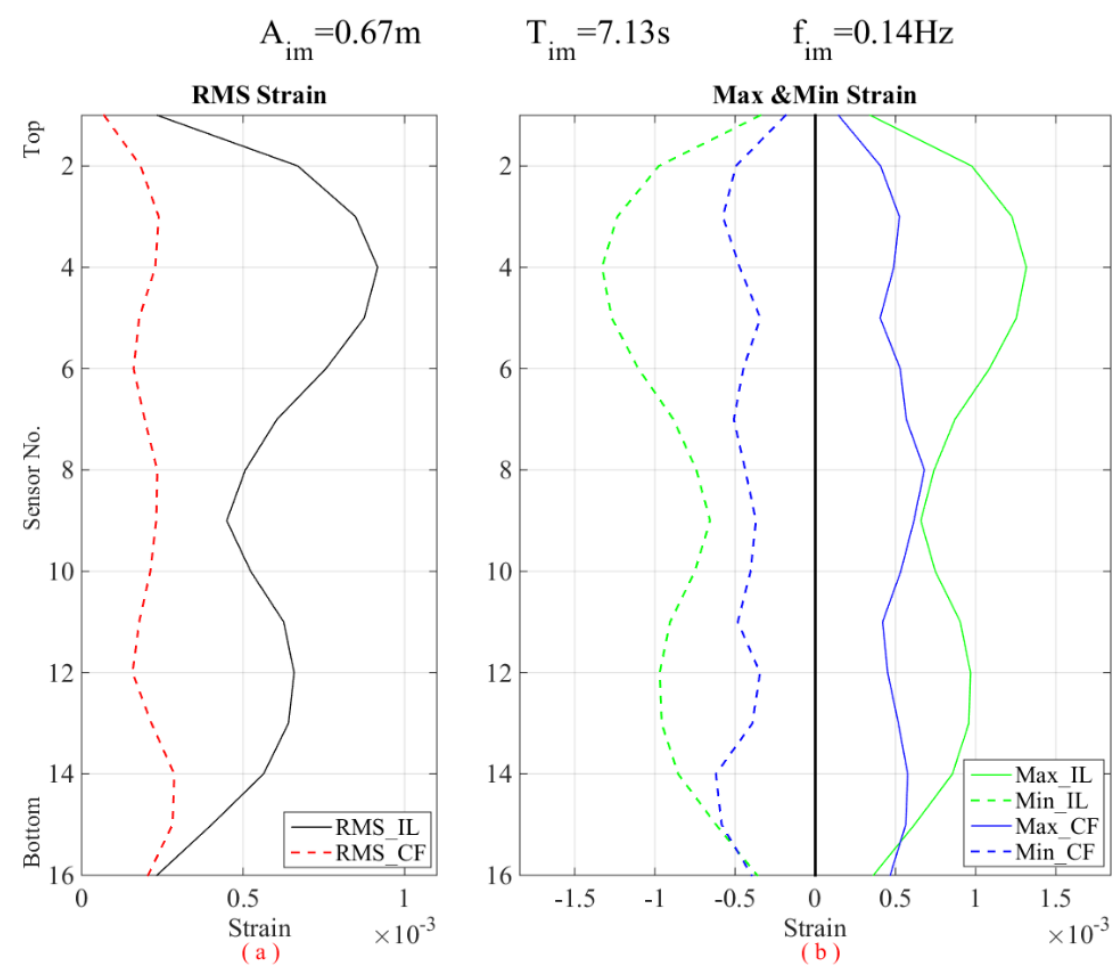

Figure 7: Statistical results of the measured strain for the case $\left(A_{i m}=0.67 m, T_{i m}=7.13 s\right)(a$ : comparison between in-plane and out-of-plane RMS strain; b: comparison between in-plane and out-of-plane maximum/ minimum strain)

Figure 8 presents the out-of-plane VIV displacement along the entire riser based on the motion reconstruction method. The maximum displacement is around 0.5D. Besides, the distribution of RMS A/D shows consistency with that of the strain, indicating the dominance of mode 4 . 


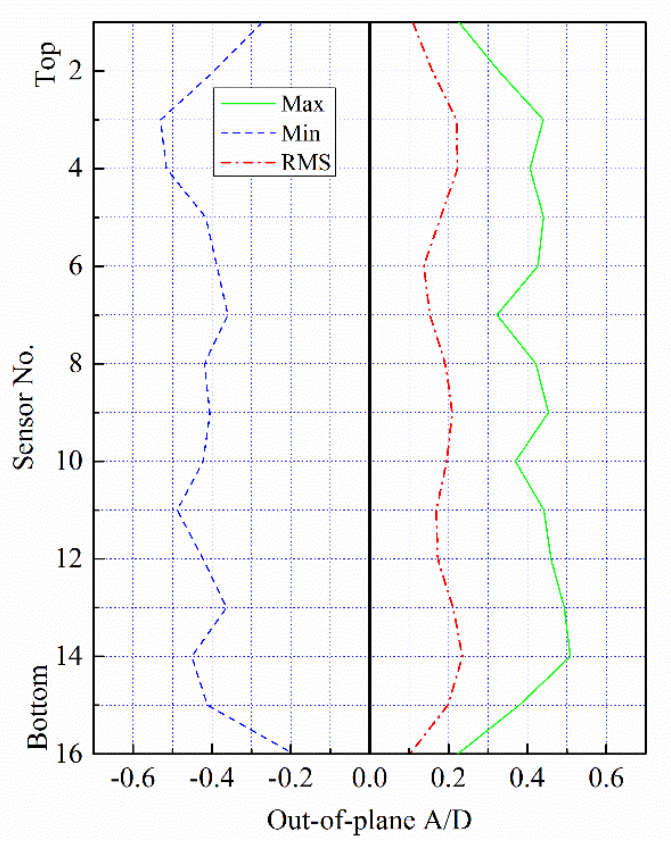

Figure 8: Reconstructed out-of-plane displacement for the case $\left(A_{i m}=0.67 m, T_{i m}=7.13 s\right)$

\subsection{Measured WIR Time-varying Responses}

Figure 9 presents contour plots showing in-plane and out-of-plane bending strain distribution for 10 vessel motion periods (there are 20 periods for each test run, we selected the $5^{\text {th }}$ to the $15^{\text {th }}$ periods in Figure 9). The color of the plot indicates the instantaneous strain amplitude. For example, red indicates a strong positive strain (top of the color bar), whereas green (bottom of the color bar) indicates a strong negative strain.

Figure 9(a) presents the vessel motion velocity time history, and Figure 9(b) illustrates the time-variation of the top axial tension around the static value which is about $32 \mathrm{Kg}$. It can be observed that the top axial tension is at the same motion period as the top motion velocity, but has an obvious phase shift. This is because the tension is not only related to the drag force in phase with velocity, but also related to the inertial force in phase with acceleration.

In Figure 9(c), the measured in-plane strain is quite periodic and it has the similar distribution as the calculated in-plane equivalent current velocity in Figure 6(b). However, in Figure 9(d), the out-of-plane strain is not as stable as the in-plane response. It can be seen that it is a typical mode 4 standing wave behavior in the first 5 periods from $41.4 \mathrm{~s}$ to $77.1 \mathrm{~s}$, but more modes participate into the whole vibration in the next 
time window and exhibits obvious travelling waves, as illustrated by the arrows in Figure 9(d). Therefore, we divide the results in Figure 9 into two time segments (each segment consists 5 motion periods) to study the section trajectory and time-frequency features.

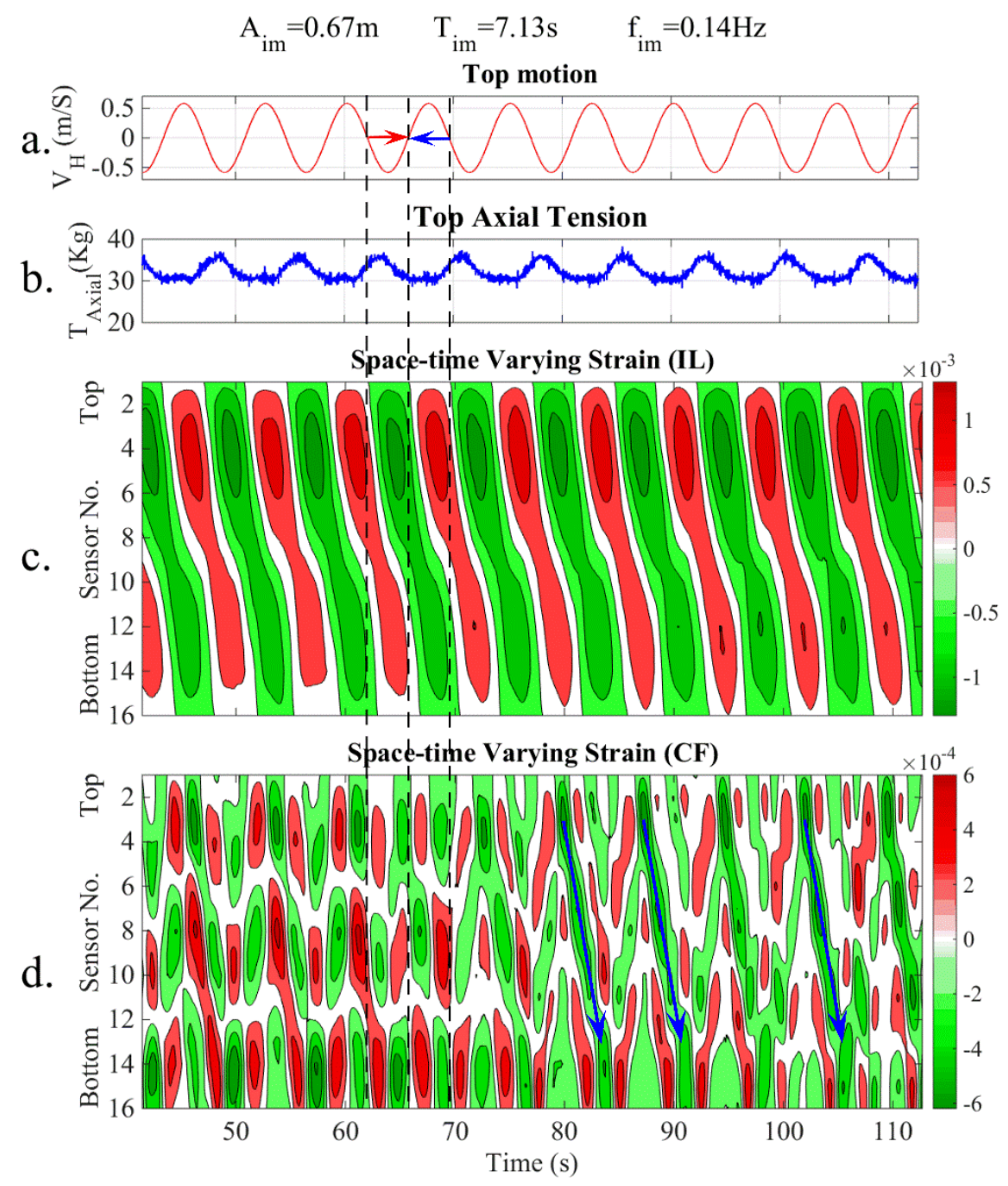

Figure 9: Space-time varying strain for the case $\left(A_{i m}=0.67 m, T_{i m}=7.13 s\right)$ (a: top motion velocity; $b$ : top axial tension variation; $c$ : measured in-plane space-time varying strain; $d$ : measured out-ofplane space-time varying strain)

\subsection{Measured WIR Response Frequency and Section Trajectories}

Figure 10 illustrates the in-plane and out-of-plane response frequency and the crosssection displacement trajectories for the first 5 selected stations from $41.4 \mathrm{~s}$ to $77.1 \mathrm{~s}$. As mentioned that there were no direct displacement measurements during the model test, therefore, the displacements trajectory is plotted using the calculated in-plane global 
motion displacement $\left(C_{D}=1.8\right)$ against the out-of-plane VIV displacement based on motion reconstruction method.

Based on the comparison between Figure 10(a) and Figure 10(b), we can see that the in-plane response frequency is the same as the top excitation frequency at $0.14 \mathrm{~Hz}$, but the out-of-plane frequency is obviously dominated by twice of the in-plane frequency at $0.28 \mathrm{~Hz}$ (simplified as $2 f_{\text {im }}$, this simplified expression for the response frequency will be used in the following text). There are also some visible but minor harmonics at $f_{\text {im }}$ and $3 f_{\text {im }}$ components. This frequency relationship is further confirmed by the 'eight' shaped trajectory at five different stations from Figure 10(c) to Figure $10(\mathrm{~g})$. It is obvious that the five trajectories are a bit different in shape. The trajectory discrepancies at different measuring stations are related to the response amplitude differences (as shown in Figure 8) and also the phase difference along the riser (phase between inplane and out-of-plane responses). It should be noted that this phase difference is an important contributor to the hydrodynamic force coefficient distribution along the riser [23].

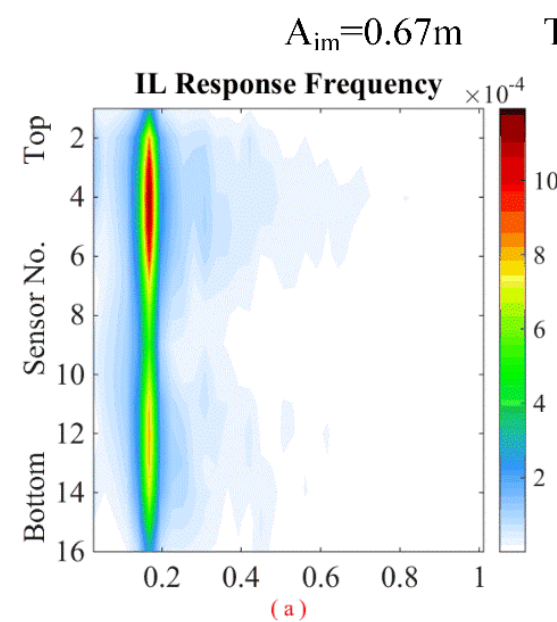

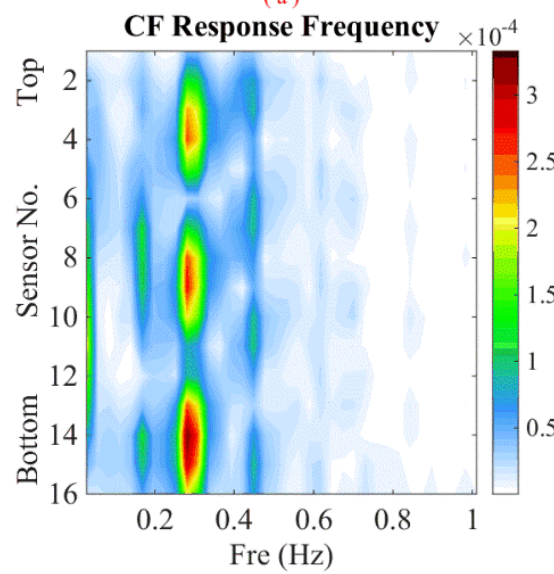

(b)

$$
\mathrm{T}_{\mathrm{im}}=7.13 \mathrm{~s} \quad \mathrm{f}_{\mathrm{im}}=0.14 \mathrm{~Hz}
$$
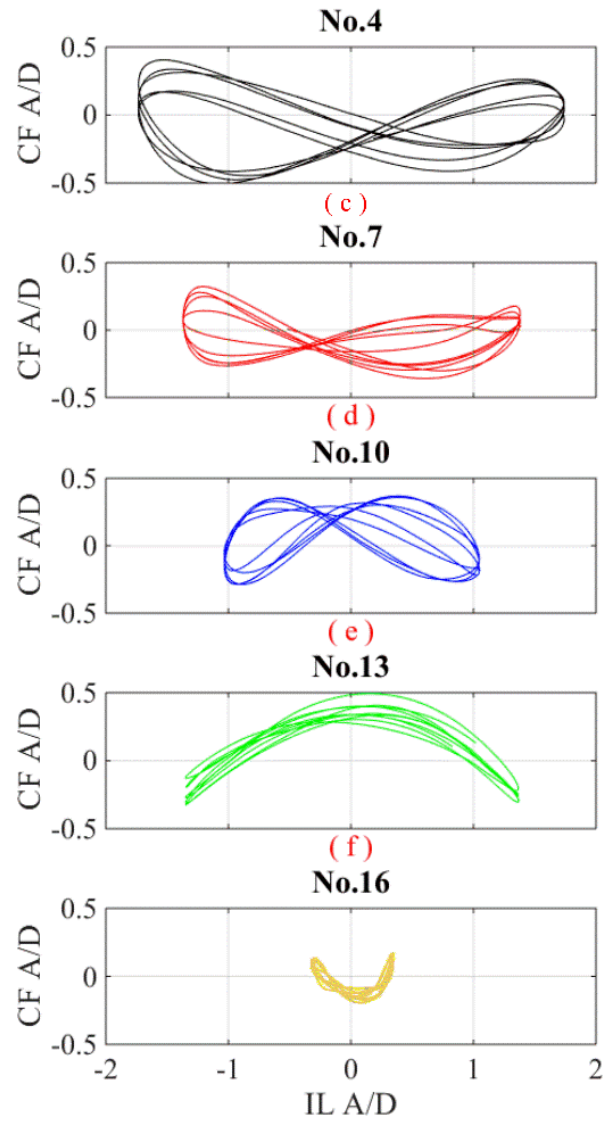

(g)

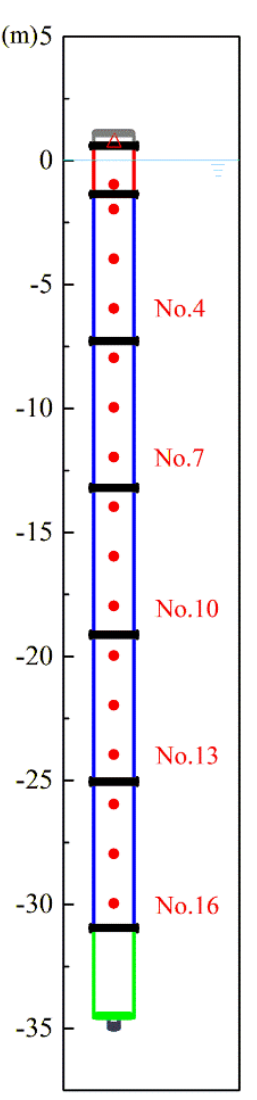


Figure 10: Response frequency and cross-section displacement trajectory for the case $\left(A_{\text {im }}=0.67 \mathrm{~m}\right.$, $T_{i m}=7.13 \mathrm{~s}$ ) from 41.4s to 77.1s (a: in-plane response frequency along the riser; b: out-of-plane response frequency along the riser; c-g: section trajectory at different station)

Figure 11 illustrates the in-plane and out-of-plane response frequency and the section trajectories for the 5 selected stations from $77.1 \mathrm{~s}$ to $112.7 \mathrm{~s}$. Different from that in the previous five periods, out-of-plane response exhibits a significant multi-mode participation as presented in Figure 11(b). In addition, trajectories for the 5 stations are also quite different from those in Figure 10. 'Three rings' (Figure 11(d)) can be clearly observed indicating the contribution from mode $5\left(3 f_{i m}\right)$ along the out-of-plane direction. Generally speaking, Figure 10 and Figure 11 are detailed presentations in different time windows for the same test case, but they demonstrate quite different out-of-plane response behavior. Inside each time window, the out-of-plane responses are quite stable and repeatable. But the response mode variation in these two time windows is supposed to be caused by both the chaotic nature of VIV and the instability of vessel motioninduced VIV under small $K C$ numbers, this is consistent with our early observation found in vessel motion-induced-VIV of a steel catenary riser [20]. 


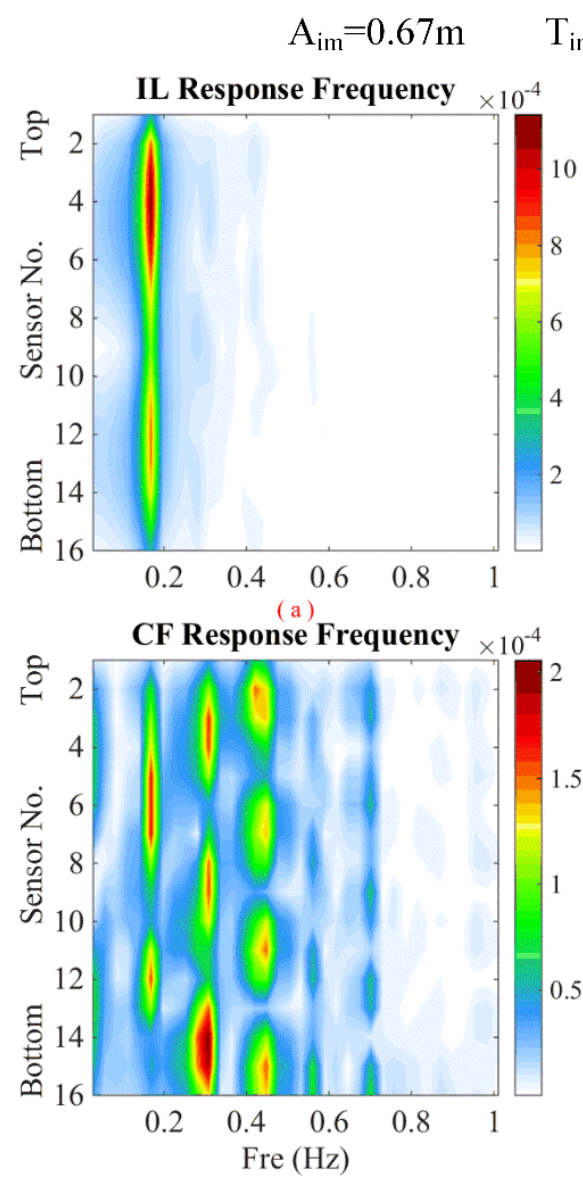

(b)

$$
\mathrm{T}_{\mathrm{im}}=7.13 \mathrm{~s} \quad \mathrm{f}_{\mathrm{im}}=0.14 \mathrm{~Hz}
$$
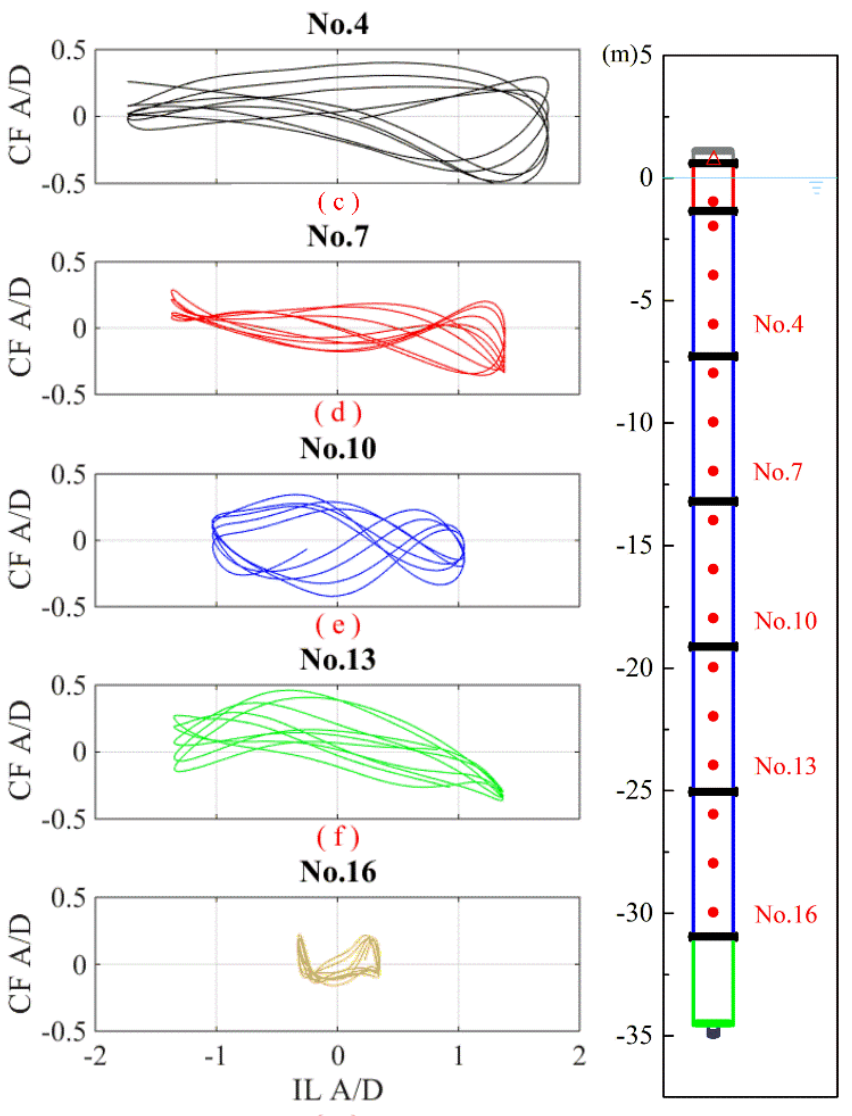

( $g$ )

Figure 11: Response frequency and section trajectory for the case $\left(A_{i m}=0.67 m, T_{i m}=7.13 s\right)$ from 77.1s to 112.7s (a: in-plane response frequency along the riser; b: out-of-plane response frequency along the riser; c-g: section trajectory at different station)

It should be further noted that there are no filtering in the whole data analysis, therefore, it can be summarized that the in-plane riser motion is completely dominated by the top excitation frequency. There might be some higher frequency components by in-plane VIV responses, but they are negligible compared to the dominant frequency component as demonstrated in the frequency contour plots in Figure 10(a) and Figure 11(a).

\subsection{Measured WIR Strain Distribution in Time-frequency Space}

Figure 12 and figure 13 further demonstrate the time-varying response frequency distribution at five selected stations separately for the two different time windows. The first row of the figure is the result for the top motion, followed by 3 columns of results including strain time histories, in-plane time-varying response frequency and out-ofplane response frequency from the left to the right. The color in the contour plots 
represents the instantaneous strength of the signal. It is stronger when the color is more close to dark red. The dominant frequencies are recognized by the black curves that follow the frequency components with the strongest strain at individual moments.

Figure 12(c) shows that the response frequency for the top tension is quite consistent with time, mainly at the top excitation frequency $f_{i m}$. There is also a visible higher frequency component $2 f_{\text {im }}$ which is supposed to be caused by the out-of-plane vibration. Figure 12(e, h, k, n, q) present the in-plane response frequency concentrated at the top motion frequency as expected. Figure 12(f, i, l, p, o, r) present the out-of-plane response frequency all at twice of the top excitation frequency at $2 f_{\text {im }}$ except for that at sensor No.7 where there is also contribution from $f_{i m}$, this is because No.7 is close to the node for mode 4 refer to Figure 10(b). Generally speaking, both in-plane global motion and out-of-plane vessel motion-induced VIV in the first 5 periods are quite time-consistent, and the dominant frequency ratio between out-of-plane and in-plane responses is 2 . 


$$
\mathrm{A}_{\mathrm{im}}=0.67 \mathrm{~m} \quad \mathrm{~T}_{\mathrm{im}}=7.13 \mathrm{~s} \quad \mathrm{f}_{\mathrm{im}}=0.14 \mathrm{~Hz}
$$
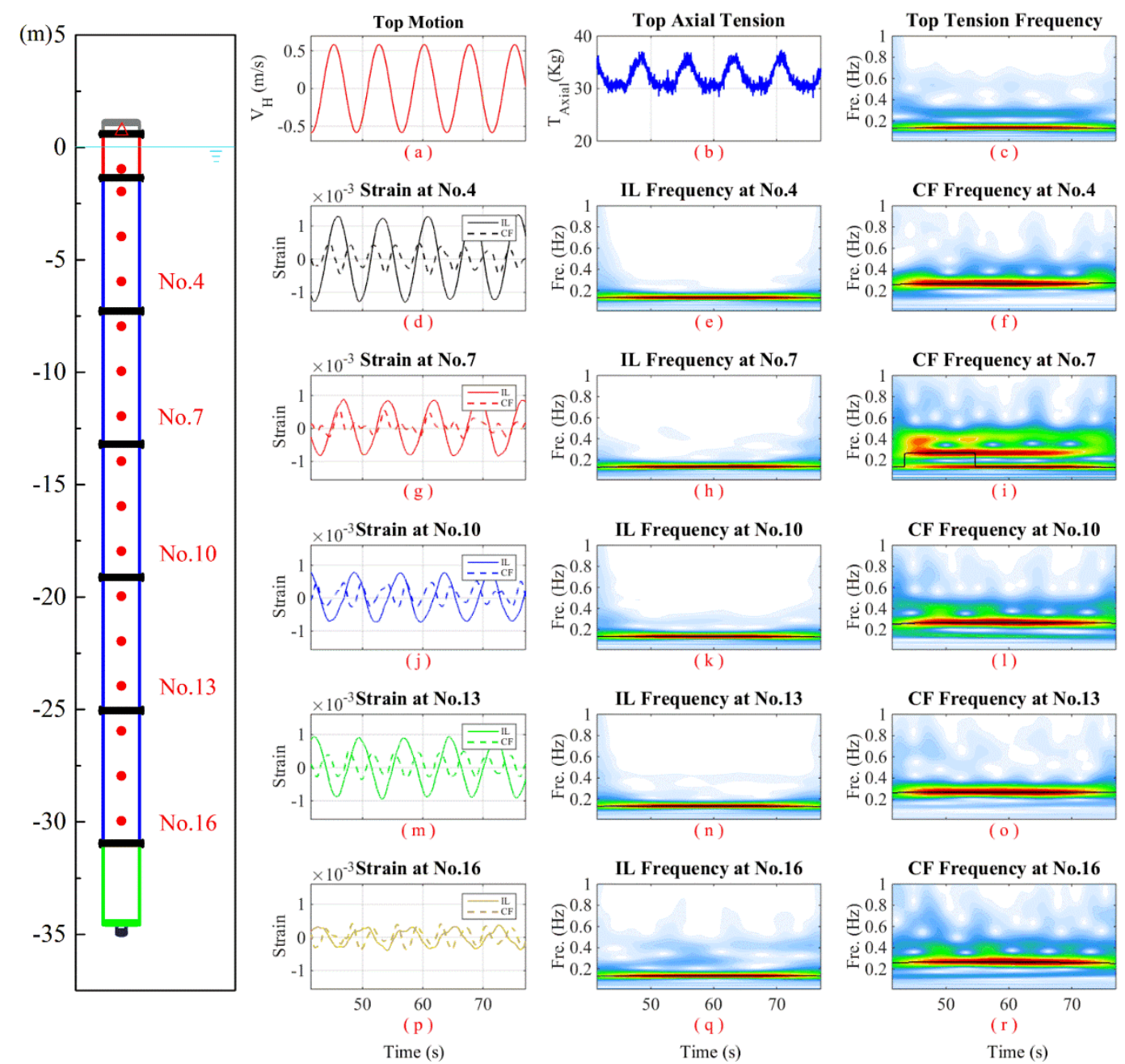

Figure 12: Time-frequency results for the case $\left(A_{i m}=0.67 m, T_{i m}=7.13 \mathrm{~s}\right)$ from $41.1 \mathrm{~s}$ to $77.1 \mathrm{~s}$ (a: top motion velocity; b: top axial tension variation; c: time-varying response frequency for the top tension; d, g, j, m, p: in-plane and out-plane bending strain time histories at 5 selected stations; e, h, k, n, q: time-varying response frequency of the in-plane responses at 5 selected stations; $f$, i, l, p, o, r: time-varying response frequency of the out-of-plane responses at 5 selected stations)

Similarly, in Figure 13, the in-plane response frequencies remain the same as the top excitation frequency at $f_{i m}$, but the out-of-plane frequencies become less regular and are more complicated compared to those in Figure 12. Out-of-plane VIV responses exhibit a distinct multi-mode behavior with discrete frequency at $f_{i m}, 2 f_{i m}$, and $3 f_{i m}$ simultaneously, this multi-mode response behavior is also seen in Figure 9(d). If we assume there is only one instantaneous dominant frequency as marked by the black 
lines on the contour plots, we can see some minor dominant frequency jumping particularly at sensor No.4, No. 7, No. 13 and No.16 as marked in Figure 13(f, i, o, r). However, the frequency distributions are still less time-varying compared to those for compliant risers under vessel motion with relatively large $K C$ numbers [20].

The reason for the relatively stable out-of-plane response frequency distribution for the WIR is partly caused by the small $K C$ number effect: the vortices from the previous motion period are still active during the next period, which may also contribute to the steady response for the small $K C$ number cases.

$$
\mathrm{A}_{\mathrm{im}}=0.67 \mathrm{~m} \quad \mathrm{~T}_{\mathrm{im}}=7.13 \mathrm{~s} \quad \mathrm{f}_{\mathrm{im}}=0.14 \mathrm{~Hz}
$$
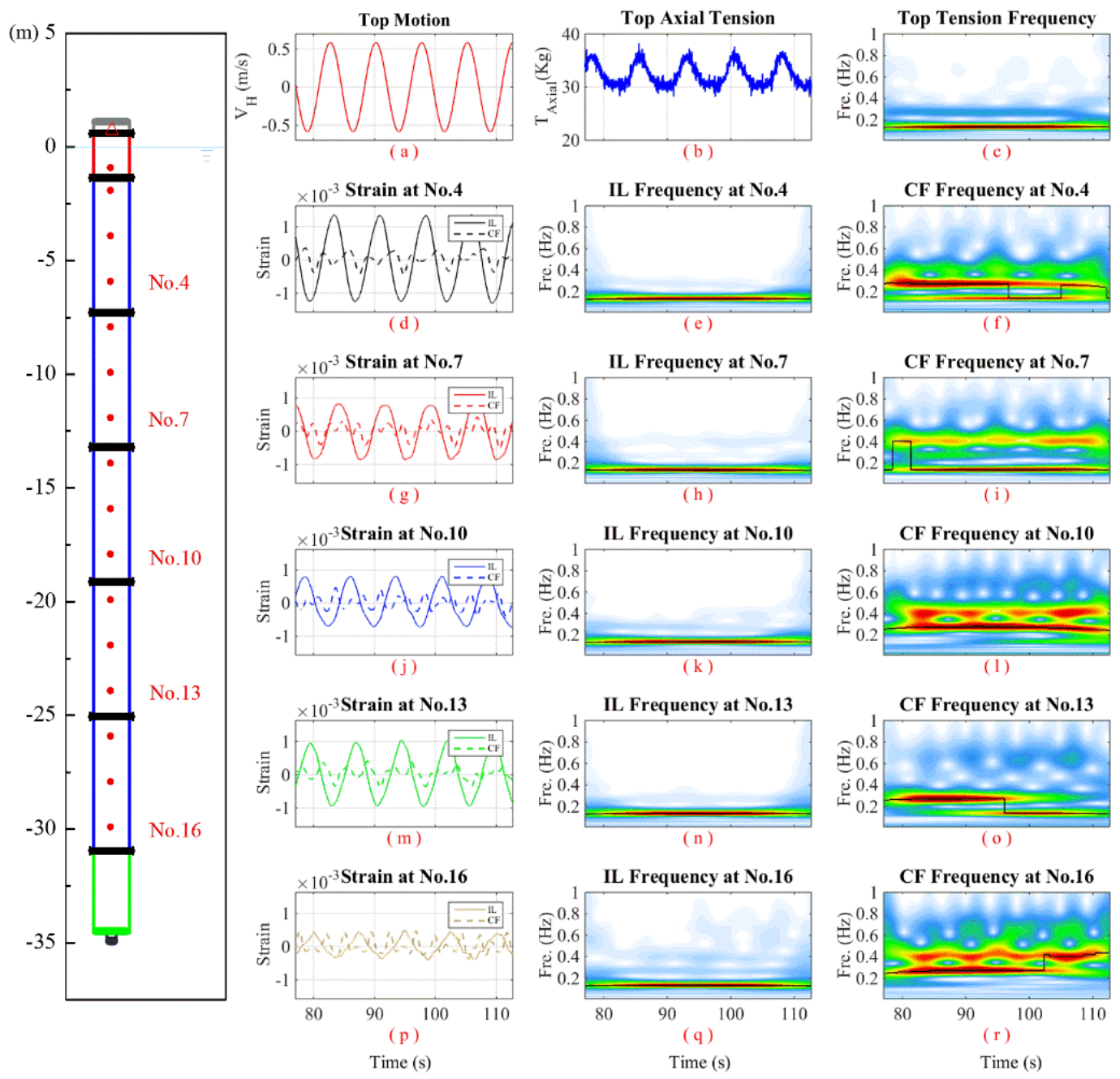

Figure 13: Time-frequency results for the case $\left(A_{i m}=0.67 m, T_{i m}=7.13 \mathrm{~s}\right)$ from $77.1 \mathrm{~s}$ to $112.7 \mathrm{~s}$ (a: top motion velocity; b: top axial tension variation; b: top tension variation; c: time-varying response frequency for the top tension; $\mathrm{d}, \mathrm{g}, \mathrm{j}$, $\mathrm{m}$, p: in-plane and out-plane bending strain time histories at 5 selected stations; e, $h, k, n$, q: time-varying response frequency of the in-plane responses at 5 
selected stations; f, i, l, p, o, r: time-varying response frequency of the out-of-plane responses at 5 selected stations)

\subsection{General discussion}

Based on the detailed case study, out-of-plane vessel motion-induced VIV is found to be significant but different from those reported for compliant risers under large $K C$ numbers. More generalized conclusions for all the 5 selected test cases are summarized as follow:

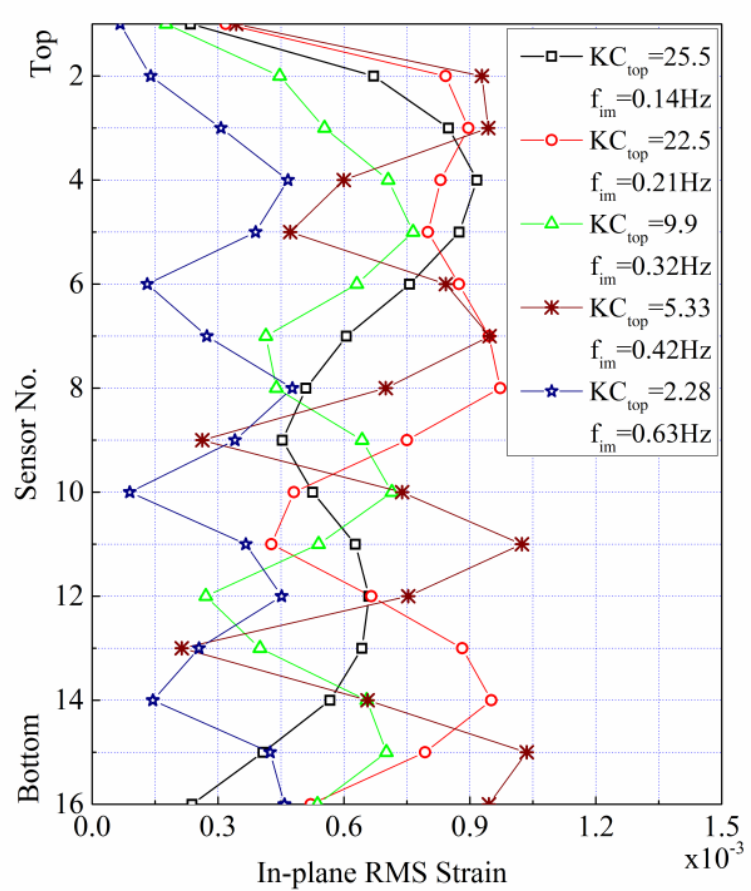

(a)

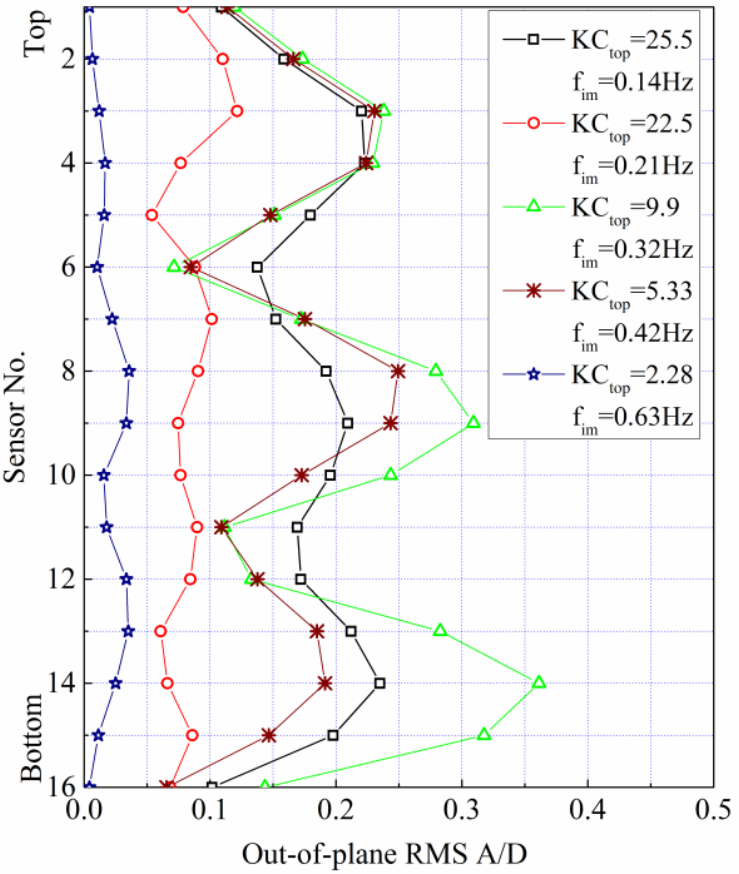

(b)

Figure 14: General results for the 5 selected cases (a. in-plane RMS strain comparison among 5 cases; b. out-of-plane RMS A/D comparison among 5 cases)

Figure 14(a) presents the measured in-plane RMS strain comparison for the five selected test cases with different top $K C$ numbers, they are all within the range between $3 \times 10^{-4}$ and $1 \times 10^{-3}$. But it should be noted that the top excitation frequencies and the maximum top excitation velocities are also quite different among these five cases, which will lead to different in-plane response modes. Therefore, in-plane RMS strain results among these 5 cases are not completely comparable. Figure 14(b) presents the 
out-of-plane RMS A/D for the five cases, which indicates two major observations:

1) The RMS A/D for the case when $K C_{\text {top }}=2.28$ is rather small at around 0.04 , the outof-plane vibration in this case is therefore negligible. The rest 4 cases have considerable out-of-plane vibration, this suggests that vessel motion-induced vibration can occur to the small $K C$ number cases even when the $K C$ number is as small as 5 . This agrees with Sumer's conclusion on flow regimes (as a function of $K C$ number) of a stationary cylinder in oscillatory flow that that alternative lift forces occur when $K C>4$. This is because asymmetry attached vortices appear when $4<K C<7$, but no vortices are shed. When $K C$ number is greater than 7, there are vortices shedding behind the cylinder [24]. 2) Different dominant modes for out-of-plane responses can be observed in Figure 14(b). This is related to both the top excitation frequency, riser natural frequencies and $K C$ number. The relationship among them will be discussed in the following paragraphs. For a rigid cylinder free to vibrate transversely in oscillatory flow, Sumer [24, 25] and Fernandes $[18,26,27]$ found the integral relationship between the out-of-plane VIV response frequency and the oscillatory flow frequency, as stated in Eq. (12). In other words, there are integral vortices shedding for each motion period.

$$
N=\frac{f_{\text {domi }}}{f_{\text {osc }}}=\frac{f_{\text {domi }}}{f_{\text {im }}}
$$

where $N$ is the frequency ratio between the out-of-plane VIV response frequency and in-plane forced motion frequency; $f_{\text {domi }}$ is the dominant frequency of out-of-plane VIV in oscillatory flow (Sumer assumed that the response frequency was consistent with time, it's similar in this paper as has been explained in Figure 12 and Figure 13, but for the compliant risers under large $K C$ number equivalent oscillatory flow, we have shown that the dominant frequency also vary with time [20]); $f_{\text {osc }}$ is the oscillatory flow frequency, which is equal to in-plane forced motion frequency $f_{i m}$ in this paper. To the WIR in this paper, the local $K C$ number varies along the riser, as has been shown in Figure 5(b). However, due to the structural continuity and spanwise correlation, the riser vibration will be in the form of one or multiple mode vibrations, as shown in Figure 7 and Figure 8. To figure out the frequency relationship between the out-of-plane and in-plane responses, frequency spectrums in Figure 10 and Figure 11 are evaluated. 
It is obvious in Figure 10(b) and Figure 11(b) that there are discrete response frequencies, and they are all corresponding to the riser natural frequencies based on the contour distribution along the riser (these response frequencies are not exactly the same in values as shown in Figure 4. This is because we use a constant added mass coefficient $C a=1$ to estimate the natural frequencies in still water, but when the riser starts to vibrate, different added mass coefficient may apply and therefore affect the real natural frequencies). Generally speaking, it can be seen that mode 4 is the dominant mode for the first five motion period in Figure 10(b) and mode 3, 4, 5 are almost equally important for the next five motion periods in Figure 11(b). These different natural modes contribution in the next five periods is assumed to be related to the $K C$ number variation along the riser. Different $K C$ number is known to lead to lift forces with different frequencies, and the spanwise correlation will further cause the whole riser to vibrate in a multi-mode response. This is quite similar to the multi-mode behavior for VIV under highly sheared current.

At each measuring station, by picking out the frequency component with the maximum strain amplitude contribution, we can obtain the dominant frequency at each location. Then the relationship between the local $K C$ number and the local out-of-plane VIV response frequency can be summarized, as listed in Table 4. We also list the rigid cylinder results by Sumer [24] and Fernandes [18] in Table 4 for comparison to better understand this $K C$ number effects on frequency ratio. It should be mentioned that Sumer and Fernandes also found out that the range of reduced velocity and Reynolds number will play an important role in determining the frequency ratio. The frequency ratio $N$ decreases with the increasing reduced velocity, and $N$ decreases at a larger reduced velocity when Reynolds number is smaller.

The local reduced velocity and Reynolds number are defined in Eq. (13):

$$
\begin{gathered}
U_{R}(s)=\frac{\max \left(V_{n}(t, s)\right)}{f_{\text {domi }}(s) \cdot D} \\
\operatorname{Re}(s)=\frac{\max \left(V_{n}(t, s)\right) \cdot D}{v}
\end{gathered}
$$

where $U_{R}(s)$ is the local reduced velocity, $V_{n}(t, s)$ is the local equivalent oscillatory current velocity, $D$ is the out diameter of the WIR, $f_{\text {domi }}(s)$ is the local dominant frequency, $\operatorname{Re}(s)$ is the local Reynolds number. 
Table 4: Summarized frequency relationship for the case $\left(A_{i m}=0.67 m, T_{i m}=7.13 s\right)$

\begin{tabular}{ccccccccc}
\hline $\begin{array}{c}\text { Station } \\
\text { No. }\end{array}$ & $\boldsymbol{K C}(\boldsymbol{s})$ & $\begin{array}{c}\boldsymbol{f}_{\text {im }} \\
(\boldsymbol{H z})\end{array}$ & $\begin{array}{c}\boldsymbol{f}_{\text {domi }} \\
(\boldsymbol{H z})\end{array}$ & $\boldsymbol{U}_{\boldsymbol{R}}(\mathbf{s})$ & $\boldsymbol{R e}(\boldsymbol{s})$ & $\begin{array}{c}N \\
\left(f_{\text {domi }} / f_{\text {im }}\right)\end{array}$ & $\begin{array}{c}\boldsymbol{N} \\
(\text { Sumer })\end{array}$ & $\begin{array}{c}\boldsymbol{N} \\
(\text { Fernandes })\end{array}$ \\
\hline Top & $\mathbf{2 5 . 5}$ & $\mathbf{0 . 1 4}$ & & & $\mathbf{9 7 3 5 0}$ & $\sim \mathbf{5}$ & $\sim \mathbf{6}$ & $\sim \mathbf{5}$ \\
end & & & & & & & & \\
1 & 18.32 & 0.14 & 0.42 & 6.12 & 69989 & 3 & 3 & 2 \\
4 & 9.42 & 0.14 & 0.28 & 5.34 & 40711 & 2 & 2 & 1 \\
7 & 8.52 & 0.14 & 0.14 & 8.74 & 33313 & 1 & 2 & 1 \\
10 & 5.73 & 0.14 & 0.28 & 3.03 & 23121 & 2 & 1 & 1 \\
13 & 8.21 & 0.14 & 0.28 & 3.99 & 30447 & 2 & 2 & 2 \\
16 & 1.9 & 0.14 & 0.28 & 1.21 & 9230 & 2 & 0 & 0 \\
\hline \hline
\end{tabular}

It should be mentioned that the local Reynolds numbers we calculated in Table 4 is in the same range as those in the rigid cylinder tests by Sumer and Fernandes. We have also considered the local reduced velocity range to pick out the corresponding $N$ from the rigid cylinder test results by Sumer and Fernandes.

Based on the comparison in Table4, we can see some differences and similarities for the frequency ratio between our flexible WIR and rigid cylinder model tests. The difference is that the local response of the WIR will be affected by the riser responses at nearby locations (spanwise correlation), this is seen in the discrepancy on $N$ mainly at station No.16. The similarity is that considering the WIR has a local $K C$ number ranging from 1.9 to 25.5(based on Figure 5(b)), and this allows the WIR to have a frequency ratio from 1 to 6 according to Sumer's conclusion (1 5 according to Fernandes). From Figure 11(b), there are clearly multi-frequency components at $f_{\text {im }}, 2 f_{\text {im }}$, $3 f_{i m}, 4 f_{i m}$ and $5 f_{i m}$, it is assumed that this $5 f_{i m}$ frequency component is contributed by the out-of-plane vibration at the top of the WIR where large local $K C$ number occurs. In this sense, the frequency ratio ranges observed from the WIR model test agree with rigid cylinder model test.

Generally speaking, most part of the riser sections have a local $K C$ number around 8 9 according to Figure 5(b), and this is in fact in agreement with the frequency spectrums in Figure 10(b) and Figure 11(b) that mode 4 contributes the most to the entire riser response. Therefore, we introduce a general frequency spectrum to find the dominant 
frequency for each case. The general frequency spectrum is defined as

$$
F(\omega)=\sum_{i=1}^{16} \hat{f}_{i}(\omega)
$$

where $\hat{f}_{i}(\omega)$ is the FFT result at the $i^{\text {th }}$ measuring station, and it is a function of strain amplitude with respect to response frequency. $F(\omega)$ is the general amplitudefrequency spectrum by summing the strain amplitude at the same frequency component for all of the 16 measuring stations.

A general frequency spectrum and the corresponding dominant frequency is illustrated by Figure 15. From the general frequency spectrum, there are three obvious frequency peaks, at $0.14 \mathrm{~Hz}\left(f_{\text {im }}\right), 0.28 \mathrm{~Hz}\left(2 f_{\text {im }},\right)$ and $0.42 \mathrm{~Hz}\left(3 f_{\text {im }}\right)$ respectively, contributions from $4 f_{i m}$ and $5 f_{i m}$ are also seen but quite insignificant. By picking out the frequency component with the maximum contribution to the entire WIR out-of-plane vibration, we identify the dominant frequency to be $0.28 \mathrm{~Hz}$ for this case, and this leads to the frequency ratio $N=2$. Considering the local $K C$ number scatters mostly around $8 \sim 9$, the frequency ratio $N=2$ agrees with rigid cylinder model test results as well.

From the frequency relationship between out-of-plane vibration and in-plane forced motion, we see that this frequency relationship is both influenced by the top $K C$ number (this top maximum $K C$ number determines the maximum out-of-plane response frequency) and the local $K C$ number distribution (because most of the riser sections have a local $K C$ number near $8 \sim 9$, the dominant frequency is therefore determined by this local $K C$ number range). 


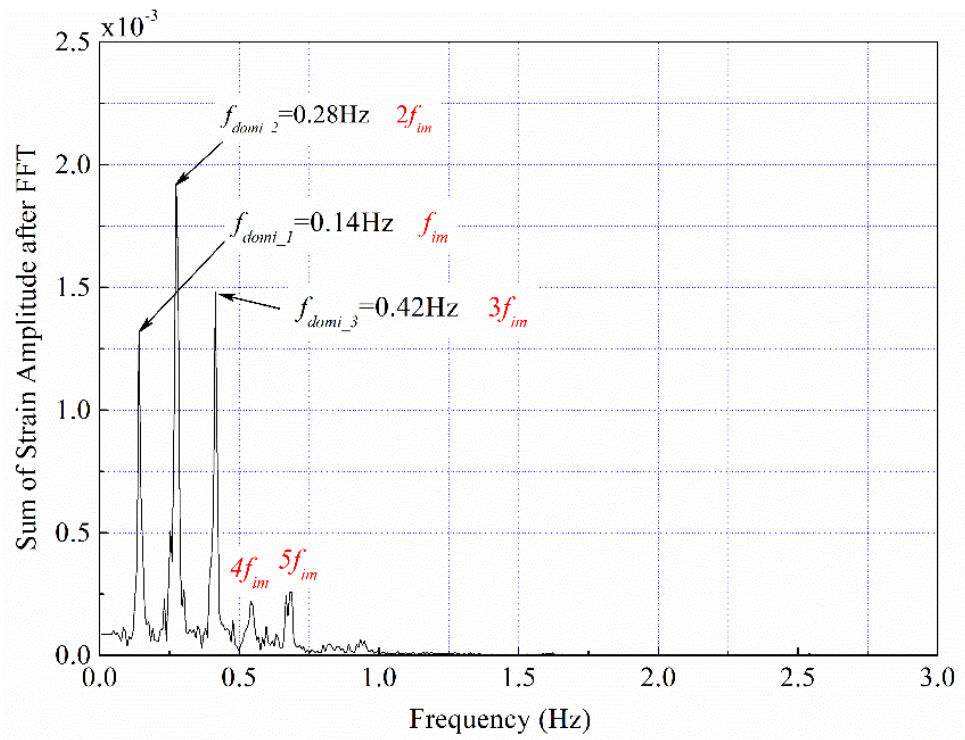

Figure 15: General stain amplitude-frequency spectrum for the case $\left(A_{\text {im }}=\mathbf{0 . 6 7 m}, T_{\text {im }}=7.13 \mathrm{~s}\right)$

Figure 16 presents the section trajectories for the other four cases, as all summarized in Table 5. In fact, the WIR section trajectory shapes are dominated by the frequency ratio $N$. Accordingly, the section trajectories vary from 'three-rings' to 'eight', 'oval' and to 'I' shape with the decreasing $K C$ number as demonstrated from the left to the right in Figure 16. The trajectory of VIV in oscillatory flow by Fernandes [18] is also listed in Table 5 for comparison, where the local reduced velocity effect has been also considered. Good agreements can be seen for the trajectory shapes of vessel motioninduced VIV of a flexible WIR and that of a rigid cylinder in oscillatory flow. This further confirms the integral relationship between out-of-plane VIV response frequency and in-plane forced motion frequency (vessel horizontal motion frequency). This frequency ratio is strongly depending on the top $K C$ number and more importantly the local $K C$ number distribution. 

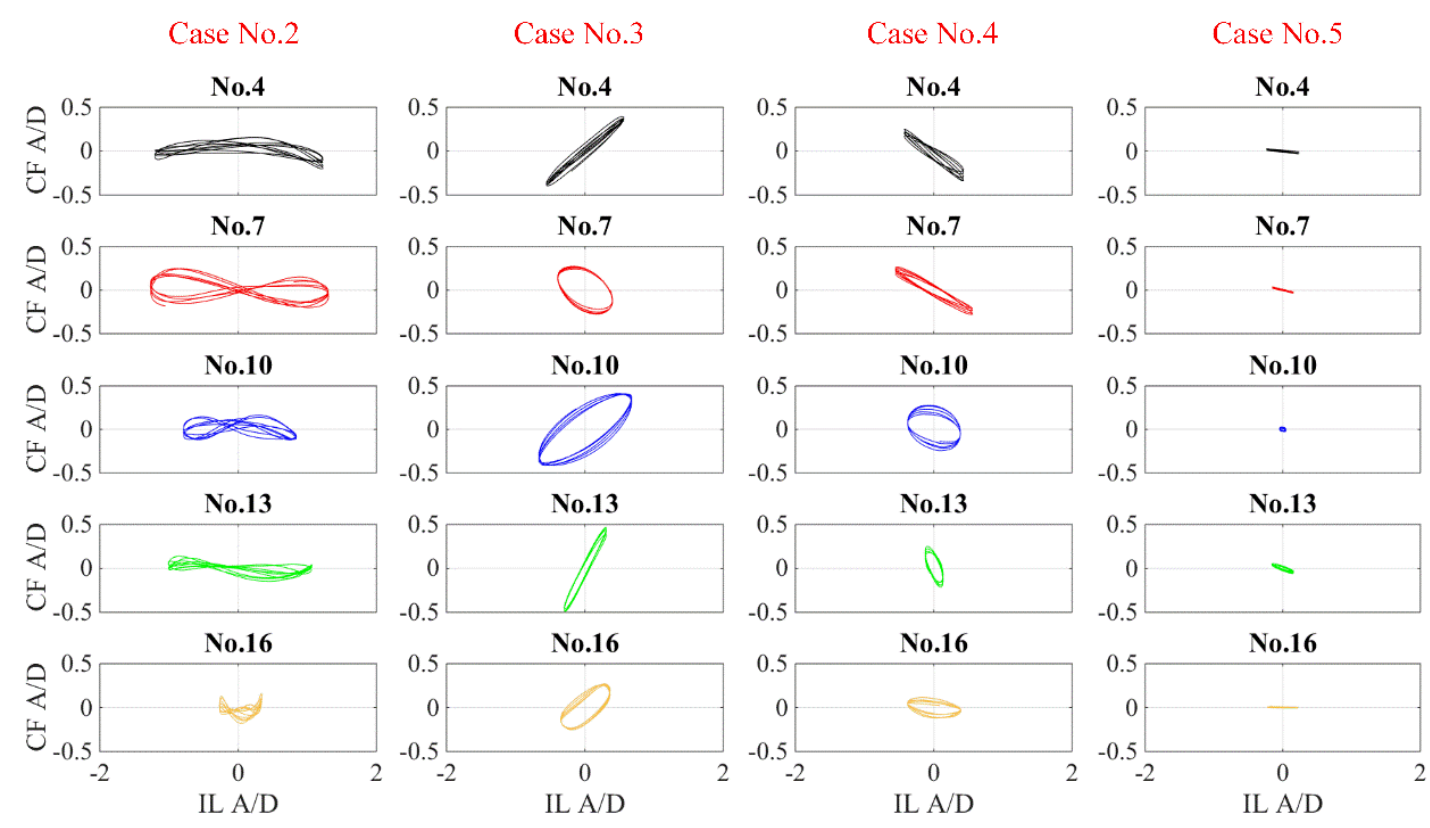

Figure 16: WIR cross-section displacement trajectories for different test cases

Table 5: Section trajectory and frequency information

\begin{tabular}{|c|c|c|c|c|c|c|c|}
\hline $\begin{array}{l}\text { Case } \\
\text { No. }\end{array}$ & $K C_{t o p}$ & $\begin{array}{c}\text { Section } \\
\text { trajectory } \\
(W I R)\end{array}$ & $\begin{array}{c}\text { Section } \\
\text { trajectory } \\
\text { (Rigid cylinder } \\
\text { by Fernandes) }\end{array}$ & $f_{\text {im }}$ & $f_{\text {domi }}$ & $N$ & $f_{\text {st_max }}$ \\
\hline 1 & 25.5 & $\begin{array}{c}\text { Three-rings; } \\
\text { Eight }\end{array}$ & $\begin{array}{c}\text { Three-rings; } \\
\text { Eight }\end{array}$ & 0.14 & 0.28 & 2 & 0.5 \\
\hline 2 & 22.5 & Eight & Eight & 0.21 & 0.42 & 2 & 0.66 \\
\hline 3 & 9.9 & Oval, I & Oval, I & 0.32 & 0.32 & 1 & 0.44 \\
\hline 4 & 5.33 & Oval, I & & 0.42 & 0.42 & 1 & 0.31 \\
\hline 5 & 2.28 & I & & 0.63 & 0.63 & 1 & 0.2 \\
\hline
\end{tabular}

What's more, discrepancy between the estimated shedding frequency and the measured dominant response frequency is quite significant, which highlights that vessel motioninduced VIV under small $K C$ number doesn't follow the Strouhal relationship by Eq. (8) using equivalent Strouhal number $S t=0.14$. This is different from the conclusions summarized from vessel motion-induced VIV for compliant risers under large $K C$ numbers [20]. It should be noted that the discrepancy shown in the estimated shedding 
frequency and the measured dominant frequency doesn't mean that vessel motioninduced VIV is not caused by vortex shedding. It only indicates that the VIV response frequency under small $K C$ number is more complex than we expect. Other shedding law may apply which is worthy of further investigation. In this sense, the $K C$ number effects on VIV dominant frequency should be well considered when developing prediction models for vessel motion-induced VIV under small $K C$ numbers.

\section{Conclusions}

In this paper, we carried out a scaled model test to evaluate the hydrodynamic performance of a free-hanging water intake riser under vessel motion. Systematic data analysis techniques were applied to investigate the riser dynamic responses. By comparing experimental and numerical results among different cases, the following conclusions could be drawn.

Owing to quite a large outer diameter, $K C$ numbers along the entire WIR under vessel motion are relatively small (smaller than 30 for all the test cases in the presented work). However, out-of-plane vessel motion-induced VIV is observed even for the case with a $K C$ number as low as 5. Results indicate these VIV responses are rather time-consistent in terms of both response amplitude and response frequency, and exhibit a standingwave behavior most of the time with a reasonable out-of-plane RMS displacement amplitude at around 0.2D. Time-varying features are also reported such as mode transition with the time evolvement. WIR section trajectories are further investigated presenting 'three-rings', 'eight', 'oval' and 'I' shapes, which confirms the integral times relationship between the out-of-plane VIV dominant frequency and local $K C$ numbers along the WIR. Moreover, discrepancy between the estimated shedding frequency and the measured dominant frequency further emphasizes the small $K C$ number effect on VIV dominant frequencies.

Most importantly, comparison between the measured and numerical simulation results suggests that there is a significant drag amplification due to the existence of the vessel motion-induced VIV. Further studies should be carried out to identify the real hydrodynamic force coefficients distribution along the WIR considering VIV and internal flow effects.

Generally speaking, results for vessel motion-induced VIV under small $K C$ numbers 
show well consistency with earlier observations for VIV of a rigid cylinder. Hopefully, these findings will be useful for gaining a better understanding on VIV induced by vessel motion, and for the development of future prediction models.

\section{Acknowledgements}

The authors gratefully acknowledge SBM offshore, USA for the permission to use these data for this publication. Support from the National Science Foundation of China (Grant No. 51279101; 51490674; 51490675) and China Scholarship Council are also greatly appreciated.

\section{Nomenclature}

\begin{tabular}{|c|c|}
\hline$D$ & Outer diameter $[m]$ \\
\hline$t$ & Thickness $[m]$ \\
\hline$E I$ & Bending stiffness $\left[\mathrm{N} \cdot \mathrm{m}^{2}\right]$ \\
\hline$E A$ & Tensile stiffness $[\mathrm{N}]$ \\
\hline$\varepsilon$ & Strain \\
\hline$T_{\text {Axial }}$ & Top axial tension $[\mathrm{N}]$ \\
\hline$A_{\text {im }}$ & Scaled top imposed motion amplitude $[\mathrm{m}]$ \\
\hline$T_{i m}$ & Scaled top imposed motion period [s] \\
\hline$f_{i m}$ & Scaled top imposed motion frequency $[\mathrm{Hz}]$ \\
\hline$K C$ & $K C$ number \\
\hline$K C_{t o p}$ & $K C$ number of the Top Vessel Motion \\
\hline $\operatorname{Re}$ & Reynolds number \\
\hline$A_{n}$ & Local oscillatory displacement amplitude [m] \\
\hline$V_{n}$ & In-plane normal velocity $[\mathrm{m} / \mathrm{s}]$ \\
\hline$S t$ & Strouhal number, 0.14 in this paper $[s]$ \\
\hline$f_{s t}$ & Estimated shedding frequency $[\mathrm{Hz}]$ \\
\hline$f_{\text {domi }}$ & Dominant response frequency $[\mathrm{Hz}]$ \\
\hline$N$ & Shedding pairs per motion period \\
\hline$U_{R}$ & Reduced velocity \\
\hline$C_{D}$ & Drag coefficient \\
\hline$C_{a}$ & Added mass coefficient \\
\hline
\end{tabular}




\section{References}

[1] White J, Longley H. FLNG technology shows promise for stranded gas fields. Offshore 2009; 69(11): 78 79.

[2] Griffin OM, Vandiver JK. Vortex-induced strumming vibrations of marine cables with attached masses. J Energ Resour Technol 1984; 106: 458-85.

[3] Lie H, Kaasen KE. Modal analysis of measurements from a large-scale VIV model test of a riser in linearly sheared flow. J Fluid and Struct 2006; 22: 557-75.

[4] Allen DW, Henning DL. Prototype vortex-induced vibration tests for production risers. In: Proc. 2001 Offshore Tech Conf, Houston, USA; 2001. OTC Paper No. 13114. [5] Tognarelli MA, Slocum ST, Frank WR, Campbell RB. VIV response of a long flexible cylinder in uniform and linearly sheared currents. In: Proc. 2004 Offshore Tech Conf, Houston, USA 2004. OTC Paper No. 16338.

[6] Chaplin JR, Bearman PW, Huera Huarte FJ, Pattenden RJ. Laboratory measurements of vortex-induced vibrations of a vertical tension riser in a stepped current. J Fluid Struct 2005; 21: 3-24.

[7] Trim AD, Braaten H, Lie H, Tognarelli MA. Experimental investigation of vortexinduced vibration of long marine risers. J Fluid Struct 2005; 21: 335-61.

[8] Vandiver JK, Marcollo H, Swithenbank S, Jhingran V. High mode number vortexinduced vibration field experiments. In: Proc. 2005 Offshore Tech Conf, Houston, USA; 2005. OTC Paper No. 17383.

[9] Baarholm GS, Larsen CM, Lie H. On fatigue damage accumulation from in-line and cross-flow vortex-induced vibrations on risers. J Fluid Struct 2006; 22: 109-27.

[10] OrcaFlex. OrcaFlex Manual: Version 9.5d. UK, 2012.

[11] RIFLEX. RIFLEX User Manual: Version 4.6.1. Marintek, Norway, 2015.

[12] Fernandes AC, Mourelle MM, Serta O, Silva S and Parra PH. Flexible and rigid risers hydrodynamic coefficients by model testing and finite element method. WIT Trans Built Env 1997; 29: 377-392.

[13] Vandiver J K. Drag coefficients of long flexible cylinders. In: Proc. 1983 Offshore Tech Conf, Houston, USA; 1983. OTC Paper No. 4490.

[14] Leira BJ, Passano E, Karunakaran D, Farnes KA, \& Giertsen E. Analysis guidelines and application of a riser-soil interaction model including trench effects. In ASME $200423^{\text {rd }}$ International Conference on Ocean, Offshore and Arctic Engineering, 
San Francisco; 2004. Paper No.OMAE2004-51527.

[15] Luis AV, Gerard CN, Platform and Environmental Parameters Data Reduction and Analysis, OTEC Cold Water Pipe At-Sea Test Program Data Analysis Project, 1985.

[16] Halkyard J, Sheikh R, Marinho T, Shi S and Ascari M. Current Developments in the Validation of Numerical Methods for Predicting the Responses of an Ocean Thermal Energy Conversion (OTEC) System Cold Water Pipe. In ASME $201433^{\text {rd }}$ International Conference on Ocean, Offshore and Arctic Engineering, San Francisco; 2014. Paper No.OMAE2014-24636.

[17] Grant RG, Litton RW, Mamidipuli P. Highly compliant rigid (HCR) riser model tests and analysis. In: Proc. 2009 Offshore Tech Conf, Houston; 2009. OTC 10973.

[18] Fernandes AC, Mirzaeisefat S, Cascão L V. Fundamental behavior of Vortex Self Induced Vibration (VSIV). Appl Ocean Res 2014, 47: 183-191.

[19] Wang J, Fu S, Baarholm R. Wu J, Larsen CM. Fatigue damage of a steel catenary riser from vortex-induced vibration caused by vessel motions. Marine Struct 2014; 39: 131-56.

[20] Wang J, Fu S, Baarholm R. Wu J, Larsen CM. Fatigue damage of a steel catenary riser from vortex-induced vibration caused by vessel motions. Ocean Eng 2015; 109: 389-400.

[21] Xiang S, Cao P, He J, Kibbee S and Bian S. Water Intake Riser Model Test and Numerical Calibration. In ASME $201534^{\text {th }}$ International Conference on Ocean, Offshore and Arctic Engineering, St. John's; 2015. Paper No.OMAE2015-42248.

[22] Cao P, He J, Xiang S. Challenges and Experiences of Model Testing a Large Deep Water Intake Riser. IBP1457_14, 2014.

[23] Wu J. Hydrodynamic force identification from stochastic vortex-induced vibration experiments with slender beams. PhD Dissertation. Norwegian University of Science and Technology, 2011.

[24] Sumer BM, Fredsøe J. Hydrodynamics around cylindrical structures (revised edition). Singapore: World Scientific Publishing Co. Pte. Ltd; 2006.

[25] Sumer BM, Fredsøe J. Transverse Vibrations of an Elastically Mounted Cylinder Exposed to an Oscillatory Flow. J. Offshore Mech. Arct. Eng 1988; 110: 387-394.

[26] Fernandes AC, Silva EMC, Franciss R, Coelho FM, Neto SFS. VSIV (vortex selfinduced vibration) kinematics, In ASME $200827^{\text {th }}$ International Conference on Ocean, Offshore and Arctic Engineering, Estoril; 2008. Paper No. 2008-57579. 
[27] Fernandes AC, Sefat SM, Cascão LV, Boas PV, Francis R. Further investigation on vortex self-induced vibration (VSIV), In ASME $201130^{\text {th }}$ International Conference on Ocean, Offshore and Arctic Engineering, Rotterdam; 2011. Paper No. 2011-50187. 